\title{
Degenerate elliptic operators in one dimension
}

\author{
Derek W. Robinson ${ }^{1}$ and Adam Sikora ${ }^{2}$
}

July 2009

\begin{abstract}
Let $H$ be the symmetric second-order differential operator on $L_{2}(\mathbf{R})$ with domain $C_{c}^{\infty}(\mathbf{R})$ and action $H \varphi=-\left(c \varphi^{\prime}\right)^{\prime}$ where $c \in W_{\text {loc }}^{1,2}(\mathbf{R})$ is a real function which is strictly positive on $\mathbf{R} \backslash\{0\}$ but with $c(0)=0$. We give a complete characterization of the self-adjoint extensions and the submarkovian extensions of $H$. In particular if $\nu=\nu_{+} \vee \nu_{-}$where $\nu_{ \pm}(x)= \pm \int_{ \pm x}^{ \pm 1} c^{-1}$ then $H$ has a unique self-adjoint extension if and only if $\nu \notin L_{2}(0,1)$ and a unique submarkovian extension if and only if $\nu \notin L_{\infty}(0,1)$. In both cases the corresponding semigroup leaves $L_{2}(0, \infty)$ and $L_{2}(-\infty, 0)$ invariant.

In addition we prove that for a general non-negative $c \in W_{\text {loc }}^{1, \infty}(\mathbf{R})$ the corresponding operator $H$ has a unique submarkovian extension.
\end{abstract}

AMS Subject Classification: 47B25, 47D06, 35J15, 31C25.

\section{Home institutions:}

1. Centre for Mathematics and its Applications

Mathematical Sciences Institute Australian National University Canberra, ACT 0200

Australia derek.robinson@anu.edu.au
2. Department of Mathematics Macquarie University

Sydney, NSW 2109

Australia

sikora@ics.mq.edu.au 


\section{Introduction}

The theory of degenerate elliptic operators on $\mathbf{R}^{d}$ displays a number of significant features which distinguishes it from the well understood non-degenerate theory. If the degeneracies are weak then there is no great difference and the degenerate theory can still be described by the techniques of the non-degenerate case, e.g. Harnack-Sobolev-Poincaré inequalities (see, for example, [Tru73] [FKS82] [FP83] [Fra91] [BM95] [FLW95] [SW06] and references therein). If, however, the degeneracies are sufficiently strong the associated diffusion process can exhibit non-ergodic behaviour; the degeneracies can spontaneously introduce barriers and obstacles to the diffusion [ERSZ07] [RS07]. These properties can lead to quite unexpected phenomena such as cloaking and invisibility [PSS06] [Wed08].

Despite the vast literature devoted to the subject many basic aspects of the degenerate theory are neither well developed nor well understood. For example, $C_{c}^{\infty}\left(\mathbf{R}^{d}\right)$ is not necessarily a core for the degenerate operator acting on $L_{2}\left(\mathbf{R}^{d}\right)$ and one has to consider boundary conditions at the barriers and obstacles. Moreover, these boundary conditions can have a substantially different nature to the classical conditions of Dirichlet, Neumann or Robin. Therefore in this paper we give a detailed analysis of the simplest situation, divergence-form operators in one-dimension. Although some of the most interesting features are not apparent in one-dimension the analysis does give a guide to possible features of the multi-dimensional case.

Let $x \in \mathbf{R} \mapsto c(x)$ be a real function in $W_{\text {loc }}^{1,2}(\mathbf{R})$ which is strictly positive on $\mathbf{R} \backslash\{0\}$. Define the second-order operator $H$ on $L_{2}(\mathbf{R})$ with domain $D(H)=C_{c}^{\infty}(\mathbf{R})$ by

$$
H \varphi=-\left(c \varphi^{\prime}\right)^{\prime}=-c \varphi^{\prime \prime}-c^{\prime} \varphi^{\prime} .
$$

Then $H$ is a positive-definite, symmetric, operator on $L_{2}(\mathbf{R})$ with range in $L_{1}(\mathbf{R}) \cap L_{2}(\mathbf{R})$. Our aim is to study the self-adjoint extensions of $H$ and the semigroups they generate. In particular we are interested in the submarkovian extensions, i.e. the extensions which generate submarkovian semigroups. One such extension always exists because the closure $\bar{h}$ of the quadratic form $h$ associated with $H$, i.e. the form

$$
h(\varphi)=(\varphi, H \varphi)=\int_{\mathbf{R}} d x c(x)\left|\varphi^{\prime}(x)\right|^{2}
$$

with domain $D(h)=C_{c}^{\infty}(\mathbf{R})$, is a Dirichlet form. Therefore the corresponding self-adjoint extension $H_{F}$ of $H$, the Friedrichs extension, is submarkovian. (For background on submarkovian semigroups and Dirichlet forms see [FOT94] [BH91] [MR92].)

If $c>0$ on the whole line then $H$ is essentially self-adjoint (see [DS63], Corollary XIII.6.15) but the situation is complicated by a degeneracy at the origin. Then there is a trichotomy of self-adjoint extensions which can be indexed by the local properties of the functions $\nu_{ \pm}$defined by

$$
x>0 \mapsto \nu_{+}(x)=\int_{x}^{1} d s c(s)^{-1} \quad \text { and } \quad x>0 \mapsto \nu_{-}(x)=\int_{-1}^{-x} d s c(s)^{-1}
$$

These functions are $H$-harmonic, i.e. $H \nu_{ \pm}=0$. The choice \pm 1 as integral limits in their definition is arbitrary since only the behaviour of the functions at the origin is important. 
Theorem 1.1 Assume $c(0)=0$.

I. If $\nu_{+} \vee \nu_{-} \notin L_{2}(0,1)$ then $H$ is essentially self-adjoint and the self-adjoint closure $\bar{H}$ of $H$ generates a submarkovian semigroup $S$ which leaves $L_{2}(-\infty, 0)$ and $L_{2}(0, \infty)$ invariant.

II. If $\nu_{+} \vee \nu_{-} \in L_{2}(0,1)$ but $\nu_{+} \vee \nu_{-} \notin L_{\infty}(0,1)$ then $H$ has a one-parameter family of self-adjoint extensions but only one extension generates a positive semigroup. This semigroup is submarkovian and leaves $L_{2}(-\infty, 0)$ and $L_{2}(0, \infty)$ invariant.

III. If $\nu_{+} \vee \nu_{-} \in L_{\infty}(0,1)$ then $H$ has a one-parameter family of self-adjoint extensions none of which leave $L_{2}(-\infty, 0)$ and $L_{2}(0, \infty)$ invariant. Moreover, there is a oneparameter subfamily of submarkovian extensions.

In particular $H$ has a unique self-adjoint extension if and only if $\nu_{+} \vee \nu_{-} \notin L_{2}(0,1)$ and a unique submarkovian extension if and only if $\nu_{+} \vee \nu_{-} \notin L_{\infty}(0,1)$.

The self-adjoint extensions of $H$ can in part be described by classical boundary conditions. In Case \all elements in the domain of the unique self-adjoint extension satisfy the condition $\left(c \varphi^{\prime}\right)\left(0_{ \pm}\right)=0$. In Case I the same conditions characterize the domain of the unique submarkovian extension. In Case III the boundary condition for a general self-adjoint extension is given by

$$
\beta\left(\left(c \varphi^{\prime}\right)\left(0_{+}\right)-\left(c \varphi^{\prime}\right)\left(0_{-}\right)\right)=\alpha\left(\varphi\left(0_{+}\right)-\varphi\left(0_{-}\right)\right)
$$

where $\alpha, \beta \in \mathbf{R}^{2} \backslash(0,0)$ and the submarkovian extensions are determined by the condition $\alpha \beta \geq 0$. The non submarkovian extensions in Case [I are exceptional. There is no comparable classification of these extensions and they do not have any obvious probabilistic interpretation.

The uniqueness criteria in the last statement of Theorem 1.1 are a measure of the order of degeneracy of $c$ at the origin. If $c(x)=O\left(x^{\delta_{ \pm}}\right)$as $x \rightarrow 0_{ \pm}$then there is a unique selfadjoint extension if and only if either $\delta_{+} \geq 3 / 2$ or $\delta_{-} \geq 3 / 2$ and a unique submarkovian extension if and only if either $\delta_{+} \geq 1$ or $\delta_{-} \geq 1$. It is notable that it suffices to have a 'strong' degeneracy on one side. This one-sideness has been stressed by Weder in the context of cloaking [Wed08, Theorem 2.5. Note also that these criteria are local properties and do not depend on the behaviour of $c$ at infinity. Next we examine a different type of characterization of uniqueness.

It follows from general operator theory that $H$ has a unique self-adjoint extension, if and only if the range of $(I+H)$ is dense in $L_{2}(\mathbf{R})$. There is a similar characterization of uniqueness of the submarkovian extension by an $L_{1}$-range condition at least if the coefficient $c$ satisfies a growth estimate. Define the positive increasing functions

$$
x \geq 1 \mapsto \mu_{+}(x)=\int_{1}^{x} d s s c(s)^{-1} \quad \text { and } \quad x \geq 1 \mapsto \mu_{-}(x)=-\int_{-x}^{-1} d s s c(s)^{-1} .
$$

Then $H \mu_{ \pm}=1$. Since $H$ is a second-order elliptic operator it is both dissipative and dispersive as an operator on $L_{1}(\mathbf{R})$. (see Section (4). In particular it is $L_{1}$-closable. Then the closure generates a positive contractive semigroup on $L_{1}(\mathbf{R})$ if and only if the range of $(I+H)$ is $L_{1}$-dense. 
Theorem 1.2 Consider the following conditions.

I. $\quad(I+H) C_{c}^{\infty}(\mathbf{R} \backslash\{0\})$ is dense in $L_{1}(\mathbf{R})$.

II. $\quad(I+H) C_{c}^{\infty}(\mathbf{R})$ is dense in $L_{1}(\mathbf{R})$.

III. The operator $H$ has a unique submarkovian extension.

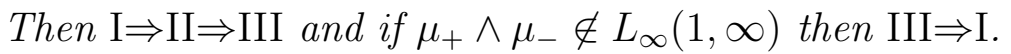

The proof of Theorem 1.1 is in two steps. First, in Section 2, we consider the analogous problems on the left and right half-lines. Secondly, in Section 3, we marry together the results for the two half-lines to obtain the description of the various extensions on the line. Our analysis on the half-line overlaps with the early work of Feller [Fel52] [Fel54] [Fel57] (see also [Man68]) but our emphasis is different and the arguments are independent. Feller classified extensions of operators acting on $L_{1}$, or on $C_{b}$, which generate positive contraction semigroups. The boundary conditions $\nu_{ \pm} \notin L_{\infty}(0,1)$ are interpretable in Feller's terminology. The condition $\nu_{+} \notin L_{\infty}(0,1)$ states that $0_{+}$is an inaccessible natural boundary (see Man68, pages 24-25).

The $L_{2}$-theory has, however, several different features not shared by the $L_{1}$-theory since there are $L_{2}$-extensions which generate continuous semigroups which do not extend to $L_{1}$ or $L_{\infty}$. Moreover, the $L_{2}$-arguments do not require any growth restrictions on $c$; there are no boundary conditions at infinity. The proof of Theorem 1.2, which is given in Section 4, is, however, based on $L_{1}$-arguments which depend in part on growth properties. The growth condition, $\mu_{+} \wedge \mu_{-} \notin L_{\infty}(1, \infty)$, coincides with Feller's criterion for $\pm \infty$ to be inacessible boundaries. The range conditions [1 and [I] in Theorem 1.2 are of independent interest as they imply that the submarkovian semigroup is conservative.

Our arguments extend to operators defined on finite intervals which are degenerate at both endpoints Ulm92 CMP98. This is briefly discussed in Section [5 where we establish the following simple statement for operators with Lipschitz continuous coefficients for which the zero set might be quite complicated.

Theorem 1.3 If $c \in W_{\mathrm{loc}}^{1, \infty}(\mathbf{R})$ is non-negative then $H$ has a unique submarkovian extension.

The proof of Theorem 1.3 uses a mixture of $L_{1^{-}}$and $L_{2}$-arguments. But these are all of a local nature and again no growth condition at infinity is necessary.

Theorems 1.2 and 1.3 should have analogues in higher dimensions.

\section{The half-line}

In this section we examine the self-adjoint extensions of the restriction $H_{+}=\left.H\right|_{C_{c}^{\infty}(0, \infty)}$ of $H$ to the right half-line. The analysis of $H_{-}=\left.H\right|_{C_{c}^{\infty}(-\infty, 0)}$ is similar.

First, since $c>0$ on $\mathbf{R} \backslash\{0\}$ the domain of the adjoint $H_{+}^{*}$ of $H_{+}$is given by

$$
D\left(H_{+}^{*}\right)=\left\{\varphi \in L_{2}(0, \infty) \cap A C_{\mathrm{loc}}(0, \infty): c \varphi^{\prime} \in A C_{\mathrm{loc}}(0, \infty),\left(c \varphi^{\prime}\right)^{\prime} \in L_{2}(0, \infty)\right\}
$$

and $H_{+}^{*} \varphi=-\left(c \varphi^{\prime}\right)^{\prime}$ for $\varphi \in D\left(H_{+}^{*}\right)$. (The details of this identification are given in [Kat80, Sections III.2.3 and III.5.5, under slightly stronger assumptions on c.) Secondly, 
the domain of the closure $\bar{H}_{+}$of $H_{+}$is obtained as the restriction of $D\left(H_{+}^{*}\right)$ by a boundary condition (see, for example, [Sto90, Theorem 10.11, [DS63, Section XIII.2 or [Far75], Section 13). In principle the boundary condition is the direct sum of a boundary value at the origin and a boundary value at infinity. But by an argument of Wintner (see [DS63], Theorem XIII.6.14) there is no boundary value at infinity. Therefore

$$
D\left(\bar{H}_{+}\right)=\left\{\varphi \in D\left(H_{+}^{*}\right): B_{+}(\varphi, \psi)=0 \text { for all } \psi \in D\left(H_{+}^{*}\right)\right\},
$$

where the boundary value $B_{+}(\cdot, \cdot)$ is a bilinear functional over $D\left(H_{+}^{*}\right)$ defined by

$$
B_{+}(\varphi, \psi)=\left(H_{+}^{*} \varphi, \psi\right)-\left(\varphi, H_{+}^{*} \psi\right)=\lim _{x \rightarrow 0_{+}}\left(\left(c \varphi^{\prime}\right)(x) \psi(x)-\varphi(x)\left(c \psi^{\prime}\right)(x)\right)
$$

for all $\varphi, \psi \in D\left(H_{+}^{*}\right)$. The limit in the boundary term exists although the limits of the individual terms in the expression do not necessarily exist. In the sequel we write $\chi\left(0_{+}\right)=\lim _{x \rightarrow 0_{+}} \chi(x)$ for any function $\chi$ over $\langle 0, \infty\rangle$. It is implicit in this usage that the limit does exist.

Proposition 2.1 Let $\nu_{+}$be the harmonic function defined on $\langle 0, \infty\rangle$ by (3).

I. If $\nu_{+} \notin L_{2}(0,1)$ then $H_{+}$is essentially self-adjoint.

II. If $\nu_{+} \in L_{2}(0,1)$ then

$$
D\left(\bar{H}_{+}\right)=\left\{\varphi \in D\left(H_{+}^{*}\right): \varphi\left(0_{+}\right)=0=\left(\nu_{+} c \varphi^{\prime}\right)\left(0_{+}\right)\right\} \varsubsetneqq D\left(H_{+}^{*}\right)
$$

and $H_{+}$has deficiency indices $(1,1)$.

Proof of Proposition 2.1,I] The proof relies on the following lemma.

Lemma 2.2 Assume $\nu_{+} \notin L_{2}(0,1)$. If $\varphi \in D\left(H_{+}^{*}\right)$ then $\left(c \varphi^{\prime}\right)\left(0_{+}\right)=0=\left(c \varphi \varphi^{\prime}\right)\left(0_{+}\right)$.

Proof If $\varphi \in D\left(H_{+}^{*}\right)$ then $\lim _{x \rightarrow 0_{+}}\left(c \varphi^{\prime}\right)(x)=\varepsilon$ exists and $\varphi^{\prime} \sim \varepsilon c^{-1}$ on $\langle 0,1]$. But $\varphi \notin L_{2}(0,1)$ unless $\varepsilon=0$. So $\left(c \varphi^{\prime}\right)\left(0_{+}\right)=0$,

$$
\left(c \varphi^{\prime}\right)(x)=\int_{0}^{x} d s\left(c \varphi^{\prime}\right)^{\prime}(s)=-\int_{0}^{x} d s\left(H_{+}^{*} \varphi\right)(s)
$$

and $\left(c \varphi^{\prime}\right)(x)=O\left(x^{1 / 2}\right)$ as $x \rightarrow 0_{+}$. Next we argue that $\lim _{x \rightarrow 0_{+}}\left(c \varphi \varphi^{\prime}\right)(x)$ exists.

The coefficient $c$ is strictly positive and bounded on each bounded interval $[a, b]$ with $b>a>0$. Since $\varphi \in D\left(H^{*}\right)$ one has $\varphi^{\prime} \in L_{2}(a, b)$. Hence

$$
\int_{a}^{b}\left(H_{+}^{*} \varphi\right) \varphi-\int_{a}^{b} c\left|\varphi^{\prime}\right|^{2}=\left(c \varphi \varphi^{\prime}\right)(a)-\left(c \varphi \varphi^{\prime}\right)(b) .
$$

But the limit as $a \rightarrow 0_{+}$of the left hand side exists although it is not necessarily finite. Therefore $\left(c \varphi \varphi^{\prime}\right)\left(0_{+}\right)$exists and we next argue that it is zero.

Suppose $\left(c \varphi \varphi^{\prime}\right)\left(0_{+}\right) \geq \varepsilon>0$. Thus there is a $\delta>0$ such that $\left(c \varphi \varphi^{\prime}\right)(x) \geq \varepsilon / 2$ for $x \in\langle 0, \delta]$. Moreover, $\left(c \varphi^{\prime}\right)(x)=O\left(x^{1 / 2}\right)$ as $x \rightarrow 0_{+}$by the foregoing argument. Therefore $|\varphi(x)| \geq a x^{-1 / 2}$ as $x \rightarrow 0_{+}$with $a>0$ unless $\varepsilon=0$. But then $\varepsilon$ must be zero since $\varphi \in L_{2}(0,1)$. An identical argument applies if $\left(c \varphi \varphi^{\prime}\right)\left(0_{+}\right)$is initially assumed to be negative. 
The symmetric operator $H_{+}$is essentially self-adjoint if and only if $\left(I+H_{+}\right) C_{c}^{\infty}(0, \infty)$ is dense in $L_{2}(0, \infty)$. Assume there is a $\psi \in L_{2}(0, \infty)$ such that $\left(\psi,\left(I+H_{+}\right) \varphi\right)=0$ for all $\varphi \in C_{c}^{\infty}(0, \infty)$. Then $\left|\left(\psi, H_{+} \varphi\right)\right| \leq\|\psi\|_{2}\|\varphi\|_{2}$ so $\psi \in D\left(H_{+}^{*}\right)$ and $\left(I+H_{+}^{*}\right) \psi=0$. But

$$
\int_{0}^{b}\left(H_{+}^{*} \psi\right) \psi=-\left(c \psi \psi^{\prime}\right)(b)+\int_{0}^{b} c\left|\psi^{\prime}\right|^{2} \geq-\left(c \psi \psi^{\prime}\right)(b)=-2^{-1}\left(c\left(\psi^{2}\right)^{\prime}\right)(b)
$$

because $\left(c \psi \psi^{\prime}\right)\left(0_{+}\right)=0$ by Lemma 2.2. Since $\psi$ is square-integrable $\psi^{2}$ cannot be monotone increasing. Hence the derivative of $\psi^{2}$ must take non-positive values for large $b$, i.e. there is a sequence $b_{n} \rightarrow \infty$ for which $-\left(c\left(\psi^{2}\right)^{\prime}\right)\left(b_{n}\right) \geq 0$. Then in the limit $n \rightarrow \infty$ one concludes that $\left(H_{+}^{*} \psi, \psi\right) \geq 0$. Therefore

$$
\|\psi\|_{2}^{2} \leq\left(\left(I+H_{+}^{*}\right) \psi, \psi\right)=0
$$

and so $\psi=0$. Thus the range of $\left(I+H_{+}\right)$is dense, $\bar{H}_{+}$is self-adjoint and $D\left(\bar{H}_{+}\right)=D\left(H_{+}^{*}\right)$. This completes the proof of Proposition 2.1, [.

The proof of Proposition 2.1II is based on the following three lemmas. The first does not require any special assumption on the behaviour of $\nu_{+}$.

Lemma 2.3 If $\varphi \in D\left(\bar{H}_{+}\right)$then $\left|\left(c \varphi^{\prime}\right)(x)\right| \leq x^{1 / 2}\left\|\bar{H}_{+} \varphi\right\|_{2}$ for all $x \in\langle 0,1]$. In particular $\left(c \varphi^{\prime}\right)\left(0_{+}\right)=0$.

Proof Fix $\varphi \in D\left(\bar{H}_{+}\right)$. Choose a sequence $\varphi_{n} \in C_{c}^{\infty}(0, \infty)$ such that $\left\|\varphi_{n}-\varphi\right\|_{2} \rightarrow 0$ and $\left\|H_{+} \varphi_{n}^{\prime}-\bar{H}_{+} \varphi\right\|_{2} \rightarrow 0$ as $n \rightarrow \infty$. Then $\left(c \varphi_{n}^{\prime}\right)(x)=-\int_{0}^{x} H_{+} \varphi_{n}$ and

$$
\left|\left(c \varphi_{n}^{\prime}\right)(x)-\left(c \varphi_{m}^{\prime}\right)(x)\right| \leq x^{1 / 2}\left\|H_{+}\left(\varphi_{n}-\varphi_{m}\right)\right\|_{2} .
$$

Thus $c \varphi_{n}^{\prime}$ converges uniformly on $\langle 0,1]$ to a limit $\psi$. But $c$ is strictly positive on each closed interval $I \subset\langle 0,1]$. Hence $\varphi_{n}^{\prime}$ converges uniformly on $I$ to $c^{-1} \psi$. In particular it is $L_{2}(I)$-convergent to $c^{-1} \psi$. Since $\varphi_{n}$ is $L_{2}(I)$-convergent to $\varphi$ and the maximal operator of differentiation is closed on $L_{2}(I)$ it follows that $\psi=c \varphi^{\prime}$. Therefore

$$
\left(c \varphi^{\prime}\right)(x)=\lim _{n \rightarrow \infty}\left(c \varphi_{n}^{\prime}\right)(x)=-\lim _{n \rightarrow \infty} \int_{0}^{x} d s\left(H_{+} \varphi_{n}\right)(s)=-\int_{0}^{x} d s\left(\bar{H}_{+} \varphi\right)(s) .
$$

Hence $\left|\left(c \varphi^{\prime}\right)(x)\right| \leq x^{1 / 2}\left\|\bar{H}_{+} \varphi\right\|_{2}$.

The second lemma gives control over the singularity of $\nu_{+}$.

Lemma 2.4 Assume $\nu_{+} \in L_{2}(0,1)$. Then $x^{1 / 2} \nu_{+}(x) \rightarrow 0$ as $x \rightarrow 0_{+}$.

Proof Suppose the statement is false. Then there exists a decreasing sequence $1 \geq x_{1} \geq$ $x_{2} \ldots>0$ and an $\varepsilon>0$ such that $x_{n}^{1 / 2} \nu_{+}\left(x_{n}\right) \geq \varepsilon>0$ for all $n \geq 1$. But by passing to a subsequence if necessary one may assume that $x_{n}>2 x_{n+1}$. Since $\nu_{+}(x)$ decreases with $x$ one then has

$$
\int_{0}^{1} d s \nu_{+}(s)^{2} \geq \sum_{n \geq 1} \nu\left(x_{n}\right)^{2}\left(x_{n}-x_{n+1}\right) \geq \sum_{n \geq 1} \nu_{+}\left(x_{n}\right)^{2} x_{n} / 2 \geq\left(\varepsilon^{2} / 2\right) \sum_{n \geq 1} 1
$$

which contradicts the assumption $\nu_{+} \in L_{2}(0,1)$.

Combination of the foregoing lemmas leads to the following conclusion. 
Corollary 2.5 Assume $\nu_{+} \in L_{2}(0,1)$. If $\varphi \in D\left(\bar{H}_{+}\right)$then $\left(\nu_{+} c \varphi^{\prime}\right)\left(0_{+}\right)=0$.

The final preparatory lemma is the following.

Lemma 2.6 Assume $\nu_{+} \in L_{2}(0,1)$. If $\varphi \in D\left(\bar{H}_{+}\right)$then $\varphi\left(0_{+}\right)=0$.

Proof Fix $\varphi \in D\left(\bar{H}_{+}\right)$. Again there is a sequence $\varphi_{n} \in C_{c}^{\infty}(0, \infty)$ such that $\left\|\varphi_{n}-\varphi\right\|_{2} \rightarrow 0$ and $\left\|H_{+} \varphi_{n}^{\prime}-\bar{H}_{+} \varphi\right\|_{2} \rightarrow 0$ as $n \rightarrow \infty$. But

$$
\varphi_{n}^{\prime}(x)=c(x)^{-1} \int_{0}^{x} d s\left(c \varphi_{n}^{\prime}\right)^{\prime}(s)
$$

for all $x>0$. Therefore

$$
\varphi_{n}(x)=\int_{0}^{x} d s c(s)^{-1} \int_{0}^{s} d t\left(c \varphi_{n}^{\prime}\right)^{\prime}(t)=-\int_{0}^{x} d t \nu_{x}(t)\left(H_{+} \varphi_{n}\right)(t)
$$

where $\nu_{x}(t)=\int_{t}^{x} d s c(s)^{-1}$. But $0 \leq \nu_{x}(t) \leq \nu_{+}(t)$ for all $x, t \in\langle 0,1]$ so

$$
\left|\varphi_{n}(x)\right| \leq\left\|\nu_{+}\right\|_{2}\left\|H_{+} \varphi_{n}\right\|_{2}
$$

for all $x \in\langle 0,1]$. Similarly

$$
\left|\varphi_{n}(x)-\varphi_{m}(x)\right| \leq\left\|\nu_{+}\right\|_{2}\left\|H_{+}\left(\varphi_{n}-\varphi_{m}\right)\right\|_{2}
$$

for all $x \in\langle 0,1]$. It follows that $\varphi_{n}$ converges to $\varphi$ uniformly on compact subsets of $\langle 0,1]$. But then one deduces from (10) that

$$
\varphi(x)=-\int_{0}^{x} d t \nu_{x}(t)\left(\bar{H}_{+} \varphi\right)(t) .
$$

Hence

$$
|\varphi(x)| \leq\left\|\nu_{+}\right\|_{2}\left(\int_{0}^{x} d t\left|\left(\bar{H}_{+} \varphi\right)(t)\right|^{2}\right)^{1 / 2}
$$

for all $x \in\langle 0,1]$. Therefore $\lim _{x \rightarrow 0_{+}} \varphi(x)=0$.

Proof of Proposition 2.1.II Suppose $\nu_{+} \in L_{2}(0,1)$. Then $D\left(\bar{H}_{+}\right) \subseteq D_{0}$ where

$$
D_{0}=\left\{\varphi \in D\left(H_{+}^{*}\right): \varphi\left(0_{+}\right)=0=\left(\nu_{+} c \varphi^{\prime}\right)\left(0_{+}\right)\right\}
$$

by Lemma 2.6 and Corollary 2.5. Next we prove the converse inclusion.

Fix $\varphi \in D_{0}$. If $\psi \in D\left(H_{+}^{*}\right)$ then $\lim _{x \rightarrow 0_{+}}\left(c \psi^{\prime}\right)(x)$ exists. Therefore there is a $b>0$ such that $\left|\left(c \psi^{\prime}\right)(x)\right| \leq b$ and $|\psi(x)| \leq b \nu_{+}(x)$ for all small $x$. But then

$$
\left|B_{+}(\varphi, \psi)\right| \leq b\left|\varphi\left(0_{+}\right)\right|+b\left|\left(\nu_{+} c \varphi^{\prime}\right)\left(0_{+}\right)\right|=0
$$

and so $\varphi \in D\left(\bar{H}_{+}\right)$. Therefore $D_{0}=D\left(\bar{H}_{+}\right)$. Then it follows from (5) that $D_{0}$ is a strict subset of $D\left(H_{+}^{*}\right)$. Hence $D\left(\bar{H}_{+}\right)$is a strict subset of $D\left(H_{+}^{*}\right)$ and $H_{+}$must have deficiency indices $(1,1)$.

At this stage we can prove an analogue of Theorem 1.1 for operators on the half-line. 


\section{Theorem 2.7}

I. If $\nu_{+} \notin L_{2}(0,1)$ then $H_{+}$is essentially self-adjoint and its closure generates a submarkovian semigroup.

II. If $\nu_{+} \in L_{2}(0,1)$ but $\nu_{+} \notin L_{\infty}(0,1)$ then $H_{+}$has a one-parameter family of self-adjoint extensions each of which generates a positive semigroup but only one extension, corresponding to the boundary condition $\left(c \varphi^{\prime}\right)\left(0_{+}\right)=0$, generates a submarkovian semigroup.

III. If $\nu_{+} \in L_{\infty}(0,1)$ then $H_{+}$has a one-parameter family of self-adjoint extensions characterized by the classical Dirichlet, Neuman and Robin boundary conditions. All the extensions generate positive semigroups and the positive(-definite) extensions generate submarkovian semigroups.

In particular $H_{+}$has a unique self-adjoint extension if and only if $\nu_{+} \notin L_{2}(0,1)$ and a unique submarkovian extension if and only if $\nu_{+} \notin L_{\infty}(0,1)$.

Proof Theorem 2.7] is a direct consequence of the first statement of Proposition 2.1, Since $H_{+}$is essentially self-adjoint the self-adjoint closure must coincide with the Friedrichs extension which generates a submarkovian semigroup as remarked in Section 1 .

The second and third statements of the theorem require more detailed analysis of the self-adjoint extensions of $H_{+}$. Throughout the following we assume $\nu_{+} \in L_{2}(0,1)$.

It follows from Proposition 2.1][I] that the deficiency indices of $H_{+}$are $(1,1)$. Therefore the codimension of $D\left(\bar{H}_{+}\right)$in $D\left(H^{*}\right)$ is two and $D\left(H_{+}^{*}\right)$ can be spanned by $D\left(\bar{H}_{+}\right)$and two auxiliary functions which we choose to be local solutions of the harmonic equation $H_{+}^{*} \psi=0$.

Let $\sigma_{+} \in C_{c}^{\infty}(0, \infty)$ satisfy $\sigma_{+}(x)=1$ if $x \in[0,1\rangle$ and $\sigma_{+}(x)=0$ if $x \geq 2$. (The choice of values 1 and 2 is not significant. One could equally well assume that $\sigma_{+}(x)=1$ if $x \in[0, \varepsilon\rangle$ and $\sigma_{+}(x)=0$ if $x \geq \delta$ with $0<\varepsilon<\delta$. It is only important that $\sigma_{+}$is equal to one near the origin.) Next set $\tau_{+}=\nu_{+} \sigma_{+}$. Clearly one has $\sigma_{+}, \tau_{+} \in D\left(H^{*}\right)$ and $\left(H_{+}^{*} \sigma_{+}\right)(x)=0=\left(H_{+}^{*} \tau_{+}\right)(x)$ for $x \in\langle 0,1\rangle$. But it follows from (17) that $\sigma_{+}, \tau_{+} \notin D\left(\bar{H}_{+}\right)$. In the sequel $\left(\sigma_{+}, \tau_{+}\right)$always denotes a pair of functions constructed in this manner.

Proposition 2.8 Assume $\nu_{+} \in L_{2}(0,1)$. Then $D\left(H_{+}^{*}\right)=D\left(\bar{H}_{+}\right)+\operatorname{span} \sigma_{+}+\operatorname{span} \tau_{+}$.

Proof First, by definition $D\left(\bar{H}_{+}\right)+\operatorname{span} \sigma_{+}+\operatorname{span} \tau_{+} \subseteq D\left(H_{+}^{*}\right)$.

Secondly, fix $\Phi \in D\left(H_{+}^{*}\right)$. Then $\lim _{x \rightarrow 0_{+}}\left(c \Phi^{\prime}\right)(x)=b$ exists. But if $\Psi=\Phi+b \tau_{+}$ then $\Psi \in D\left(H_{+}^{*}\right)$ and $\left(H_{+}^{*} \Psi\right)(x)=\left(H_{+}^{*} \Phi\right)(x)$ for $x \in\langle 0,1\rangle$. Moreover, $\left(c \Psi^{\prime}\right)\left(0_{+}\right)=$ $\left(c \Phi^{\prime}\right)\left(0_{+}\right)-b=0$. Then, however,

$$
\begin{aligned}
\Psi(x) & =\Psi(1)-\int_{x}^{1} d s c(s)^{-1} \int_{0}^{s} d t\left(H_{+}^{*} \Psi\right)(t) \\
& =\Psi(1)-\int_{0}^{1} d s c(s)^{-1} \int_{0}^{s} d t\left(H_{+}^{*} \Phi\right)(t)+\int_{0}^{x} d s c(s)^{-1} \int_{0}^{s} d t\left(H_{+}^{*} \Phi\right)(t)
\end{aligned}
$$

for all $x \in\langle 0,1\rangle$. Both the latter integrals are well defined since $\nu_{+} \in L_{2}(0,1)$. Moreover,

$$
\begin{aligned}
\left|\int_{0}^{x} d s c(s)^{-1} \int_{0}^{s} d t\left(H_{+}^{*} \Phi\right)(t)\right| & =\left|\int_{0}^{x} d t\left(H_{+}^{*} \Phi\right)(t) \int_{t}^{x} c(s)^{-1}\right| \\
& \leq\left\|\nu_{+}\right\|_{2}\left(\int_{0}^{x} d t\left|\left(H_{+}^{*} \Phi\right)(t)\right|^{2}\right)^{1 / 2}
\end{aligned}
$$


which establishes that

$$
a=\lim _{x \rightarrow 0_{+}} \Psi(x)=\lim _{x \rightarrow 0_{+}}\left(\Phi(x)+b \tau_{+}(x)\right)
$$

exists and $a<\infty$. Next define $\varphi$ by

$$
\varphi=\Phi-a \sigma_{+}+b \tau_{+}=\Psi-a \sigma_{+} .
$$

Then $\varphi \in D\left(H_{+}^{*}\right)$. Moreover, if $x \in\langle 0,1\rangle$ then

$$
\left(c \varphi^{\prime}\right)(x)=\left(c \Psi^{\prime}\right)(x)=-\int_{0}^{x} d s\left(H_{+}^{*} \Psi\right)(s) .
$$

Therefore $\left|\left(c \varphi^{\prime}\right)(x)\right|=O\left(x^{1 / 2}\right)$ as $x \rightarrow 0_{+}$. Hence $\left(\nu_{+} c \varphi^{\prime}\right)\left(0_{+}\right)=0$ by Lemma 2.4. But $\varphi\left(0_{+}\right)=\Psi\left(0_{+}\right)-a=0$ by the definition of $a$. Therefore $\varphi \in D\left(\bar{H}_{+}\right)$by Proposition 2.1][I], Hence $D\left(H_{+}^{*}\right) \subseteq D\left(\bar{H}_{+}\right)+\operatorname{span} \sigma_{+}+\operatorname{span} \tau_{+}$.

The one-parameter family of self-adjoint extensions of $\bar{H}_{+}$can now be specified by restricting $H_{+}^{*}$ to subspaces of $D\left(H_{+}^{*}\right)$ obtained by supplementing $D\left(\bar{H}_{+}\right)$through the addition of a one-dimensional subspace of $\operatorname{span} \sigma_{+}+\operatorname{span} \tau_{+}$.

Proposition 2.9 Assume $\nu_{+} \in L_{2}(0,1)$. Let $(\alpha, \beta) \in \mathbf{R}^{2} \backslash(0,0)$. Define

$$
D_{\alpha, \beta}=D\left(\bar{H}_{+}\right)+\operatorname{span}\left(\beta \sigma_{+}-\alpha \tau_{+}\right)=\left\{\varphi \in D\left(H^{*}\right): B_{+}\left(\beta \sigma_{+}-\alpha \tau_{+}, \varphi\right)=0\right\} .
$$

Then the restriction $H_{\alpha, \beta}$ of $H_{+}^{*}$ to $D_{\alpha, \beta}$ is a self-adjoint extension of $\bar{H}_{+}$and $H_{\alpha, \beta}=H_{\alpha^{\prime}, \beta^{\prime}}$ if and only if $\alpha \beta^{\prime}=\beta \alpha^{\prime}$.

Proof It follows by the definition of $H_{\alpha, \beta}$ that $\bar{H}_{+} \subseteq H_{\alpha, \beta} \subseteq H_{+}^{*}$. Therefore $\Phi \in D\left(H_{\alpha, \beta}^{*}\right)$ if and only if $\Phi \in D\left(H_{+}^{*}\right)$ and $B_{+}(\Psi, \Phi)=0$ for all $\Psi \in D_{\alpha, \beta}$ (see the lemma on page 86 of [Far75]). But if $\Phi=\varphi+a \sigma_{+}-b \tau_{+} \in D\left(H_{+}^{*}\right)$ and $\Psi=\psi+\lambda\left(\beta \sigma_{+}-\alpha \tau_{+}\right) \in D_{\alpha, \beta}$ with $\varphi, \psi \in D\left(\bar{H}_{+}\right)$and $a, b, \lambda \in \mathbf{R}$ then

$$
B_{+}(\Psi, \Phi)=\lambda B_{+}\left(\beta \sigma_{+}-\alpha \tau_{+}, \Phi\right)=\lambda(a \alpha-b \beta) .
$$

Thus $\Phi \in D\left(H_{\alpha, \beta}^{*}\right)$ if and only if $a \alpha=b \beta$. But then $\Phi \in D_{\alpha, \beta}$. Since $H_{\alpha, \beta}^{*} \subseteq H^{*}$ it follows that $H_{\alpha, \beta}^{*} \Phi=H_{+}^{*} \Phi=H_{\alpha, \beta} \Phi$ and consequently $H_{\alpha, \beta}$ is self-adjoint.

The definitions of $D_{\alpha, \beta}$ and $H_{\alpha, \beta}$ are clearly independent of a change $(\alpha, \beta) \rightarrow\left(\alpha^{\prime}, \beta^{\prime}\right)$ if $\alpha \beta^{\prime}=\beta \alpha^{\prime}$.

Next we prove the second statement of Theorem 2.7. This is the most complicated and the most interesting case. Throughout the sequel we use the notation $h_{+}$and $h_{\alpha, \beta}$ for the closed quadratic forms associated with the operators $H_{+}$and $H_{\alpha, \beta}$.

Theorem 2.10 Assume $\nu_{+} \in L_{2}(0,1)$ but $\nu_{+} \notin L_{\infty}(0,1)$. Then $\bar{h}_{+}=h_{0,1}$, the Friedrichs extension $H_{F}$ of $H_{+}$is equal to $H_{0,1}$ and

$$
D\left(H_{F}\right)=\left\{\varphi \in D\left(H_{+}^{*}\right):\left(c \varphi^{\prime}\right)\left(0_{+}\right)=0\right\} .
$$

The semigroup generated by $H_{F}$ is submarkovian. Moreover, $H_{F}$ is the unique selfadjoint extension of $H_{+}$which generates a submarkovian semigroup. 
A key part of the proof is the following lemma which follows from the arguments of [ERSZ07], Proposition 6.5, or [RS07], Example 3.3.

Lemma 2.11 Assume $\nu_{+} \notin L_{\infty}(0,1)$. Then $\sigma_{+} \in D\left(\bar{h}_{+}\right)$.

Proof The proof is a repetition of the arguments of [ERSZ07] but since the result is crucial for the sequel we sketch the details.

Define $\chi_{n}: \mathbf{R} \rightarrow[0,1]$ by

$$
\chi_{n}(x)= \begin{cases}1 & \text { if } x \leq n^{-1}, \\ \nu_{n}^{-1} \nu_{+}(x) & \text { if } x \in\left\langle n^{-1}, 1\right\rangle, \\ 0 & \text { if } x \geq 1\end{cases}
$$

where $n \in \mathbf{N}$ and $\nu_{n}=\nu_{+}\left(n^{-1}\right)$. Since $\nu_{+} \notin L_{\infty}(0,1)$ it follows that $\nu_{n} \rightarrow \infty$ as $n \rightarrow \infty$. Now $\chi_{n}$ is absolutely continuous, $\left\|\chi_{n} \sigma_{+}\right\|_{2} \rightarrow 0$ as $n \rightarrow \infty$ and $\chi_{n} \sigma_{+}^{\prime}=0$. Moreover,

$$
\int_{0}^{\infty} c\left|\left(\chi_{n} \sigma_{+}\right)^{\prime}\right|^{2}=\int_{n^{-1}}^{1} c\left|\chi_{n}^{\prime} \sigma_{+}\right|^{2}=\int_{n^{-1}}^{1} c\left|\chi_{n}^{\prime}\right|^{2}
$$

because $\sigma_{+}=1$ on $[0,1\rangle$. But $\chi_{n}^{\prime}=\nu_{n}^{-1} c^{-1}$ on the interval $\left[n^{-1}, 1\right]$. Therefore

$$
\int_{0}^{\infty} c\left|\left(\chi_{n} \sigma_{+}\right)^{\prime}\right|^{2}=\nu_{n}^{-2} \int_{n^{-1}}^{1} c^{-1}=\nu_{n}^{-1} .
$$

Next $\operatorname{supp}\left(1-\chi_{n}\right) \sigma_{+} \subseteq\left[n^{-1}, 1\right]$. Consequently one checks that $\left(1-\chi_{n}\right) \sigma_{+} \in D\left(\bar{h}_{+}\right)$. Now $\left\|\left(1-\chi_{n}\right) \sigma_{+}-\sigma_{+}\right\|_{2}=\left\|\chi_{n} \sigma_{+}\right\|_{2} \rightarrow 0$ as $n \rightarrow \infty$ and

$\bar{h}_{+}\left(\left(1-\chi_{n}\right) \sigma_{+}-\left(1-\chi_{m}\right) \sigma_{+}\right) \leq 2 \int_{0}^{\infty} c\left|\left(\sigma_{+} \chi_{n}\right)^{\prime}\right|^{2}+2 \int_{0}^{\infty} c\left|\left(\sigma_{+} \chi_{m}\right)^{\prime}\right|^{2}=2\left(\nu_{n}^{-1}+\nu_{m}^{-1}\right)$.

Since $\nu_{n}^{-1} \rightarrow 0$ as $n \rightarrow \infty$ it follows that $\sigma_{+} \in D\left(\bar{h}_{+}\right)$.

Proof of Theorem 2.10 It follows from Lemma 2.11 that $D\left(\bar{H}_{+}\right)+\operatorname{span} \sigma_{+} \subseteq D\left(\bar{h}_{+}\right)$. Then since $h_{0,1}(\varphi)=h_{+}(\varphi)$ for all $\varphi \in C_{c}^{\infty}(0, \infty)$ one deduces that $h_{0,1}(\Phi)=\bar{h}_{+}(\Phi)$ for all $\Phi \in D\left(\bar{H}_{+}\right)+\operatorname{span} \sigma_{+}$. Hence $h_{0,1}=\bar{h}_{+}$and $H_{0,1}=H_{F}$. The boundary condition follows since $\varphi \in D\left(H_{0,1}\right)$ requires $B_{+}\left(\sigma_{+}, \varphi\right)=0$. But one has $B_{+}\left(\sigma_{+}, \varphi\right)=\left(c \varphi^{\prime}\right)\left(0_{+}\right)$.

The form $h_{+}$is given by (2) with the integral restricted to the half-line. Then the closure $\bar{h}_{+}$is a Dirichlet form by standard estimates. Therefore the semigroup generated by $H_{0,1}$ is submarkovian. Now suppose that the semigroup $S^{\alpha, \beta}$ generated by $H_{\alpha, \beta}$ with $\alpha \neq 0$ is submarkovian. Then $h_{\alpha, \beta}$ is a Dirichlet form. In particular if $\varphi \in D\left(h_{\alpha, \beta}\right)$ is positive then $R \wedge \varphi \in D\left(h_{\alpha, \beta}\right)$ for all $R>0$ and

$$
h_{\alpha, \beta}(R \wedge \varphi) \leq h_{\alpha, \beta}(\varphi) .
$$

But it follows from the definition of $D_{\alpha, \beta}=D\left(H_{\alpha, \beta}\right)$ that $\beta \sigma_{+}-\alpha \tau_{+} \in D_{\alpha, \beta} \subseteq D\left(h_{\alpha, \beta}\right)$. Moreover $\sigma_{+} \in D\left(\bar{h}_{+}\right) \subseteq D\left(h_{\alpha, \beta}\right)$ by Lemma 2.11. Since $\alpha \neq 0$ one must then have $\tau_{+} \in D\left(h_{\alpha, \beta}\right)$. In particular $h_{\alpha, \beta}\left(\tau_{+}\right)<\infty$. Now we can apply (15) with $\varphi=\tau_{+}$. Since $R \wedge \tau_{+} \in D\left(\bar{h}_{+}\right) \subseteq D\left(h_{\alpha, \beta}\right)$ one has

$$
\bar{h}_{+}\left(R \wedge \tau_{+}\right)=h_{\alpha, \beta}\left(R \wedge \tau_{+}\right) \leq h_{\alpha, \beta}\left(\tau_{+}\right) .
$$


But $\tau_{+}=\nu_{+}$and $\tau_{+}^{\prime}=-c^{-1}$ on $\langle 0,1]$. Therefore

$$
\int_{S_{R}} d x c(x)^{-1}=\int_{S_{R}} d x c(x)\left|\tau_{+}^{\prime}(x)\right|^{2} \leq \bar{h}_{+}\left(R \wedge \tau_{+}\right)=h_{\alpha, \beta}\left(R \wedge \tau_{+}\right) \leq h_{\alpha, \beta}(\tau)<\infty
$$

where $S_{R}=\left\{x \in\langle 0,1]: \tau_{+}(x) \leq R\right\}$. But $\nu_{+} \notin L_{\infty}(0,1)$. So the supremum of the left hand side over $R$ is infinite. This is a contradiction so $h_{\alpha, \beta}$ cannot be a Dirichlet form.

Remark 2.12 If $\nu_{+} \in L_{2}(0,1)$ but $\nu_{+} \notin L_{\infty}(0,1)$ then the form $h_{0,1}$ of the Friedrichs extension $H_{0,1}$ has the integral representation $h_{0,1}(\varphi)=\int_{0}^{\infty} c\left|\varphi^{\prime}\right|^{2}$ for all $\varphi \in D\left(h_{0,1}\right)$ and the operator domain $D\left(H_{0,1}\right)$ is distinguished by the boundary condition $\left(c \varphi^{\prime}\right)\left(0_{+}\right)=0$. But the situation is quite different for the forms $h_{\alpha, \beta}$ and operators $H_{\alpha, \beta}$ if $\alpha \neq 0$. Then there is no analogue of the form representation nor of the boundary condition. For example, the foregoing argument establishes that $\tau_{+} \in D\left(h_{\alpha, \beta}\right)$. But then

$$
\int_{0}^{\infty} c\left|\tau_{+}^{\prime}\right|^{2} \geq \int_{0}^{1} c\left|\nu_{+}^{\prime}\right|^{2}=\int_{0}^{1} c^{-1}=\nu_{+}(0)=\infty
$$

Moreover, if $\Phi=\varphi+a \sigma_{+}-b \tau_{+} \in D\left(H_{\alpha, \beta}\right)$ with $\varphi \in D(\bar{H})$ one has the boundary condition $a \alpha=b \beta$. Then $b=\left(c \Phi^{\prime}\right)\left(0_{+}\right)$but one cannot identify $a$ in terms of the value of $\Phi$ and its derivatives at the origin.

Although the semigroups $S^{\alpha, \beta}$ generated by the $H_{\alpha, \beta}$ with $\alpha \neq 0$ cannot be submarkovian we next argue that they are positive. First since $H_{+}$has deficiency indices $(1,1)$ there is, for each $\gamma>0$, a unique, up to a multiplicative factor, $L_{2}$-solution of the deficiency equation $\left(\gamma I+H_{+}^{*}\right) \eta=0$. Therefore there is a unique positive, decreasing, normalized, $L_{2}$-solution $\eta_{\gamma}$. More precisely, $\eta_{\gamma}$ is non-negative and non-increasing. To establish this note that the equation has the explicit form $\left(c \eta^{\prime}\right)^{\prime}=\gamma \eta \in L_{2}(0, \infty)$ and this implies that $\eta^{\prime}$ is continuous. Hence $\eta$ is a locally $C^{1}$-function. Now suppose $\eta(a)=0$ for some $a>0$. Then $\left(c \eta \eta^{\prime}\right)(a)=0$ and it follows from (18), with $\varphi=\eta$ and $H_{+}^{*} \eta=-\gamma \eta$, that

$$
2^{-1} c\left(\eta^{2}\right)^{\prime}(b)=\left(c \eta \eta^{\prime}\right)(b)=\int_{a}^{b}\left(\gamma|\eta|^{2}+c\left|\eta^{\prime}\right|^{2}\right)
$$

for all $b \geq 0$. In particular $\left(\eta^{2}\right)^{\prime}(b) \geq 0$ for all $b \geq a$. But since $\eta$ is square integrable $\eta^{2}$ cannot be monotonically increasing. Therefore $\eta(b)=0$ for all $b \geq a$. If, however, $b \leq a$ then $\left(\eta^{2}\right)^{\prime}(b) \leq 0$. Therefore $\eta$ is either non-negative and non-increasing, or non-positive and non-decreasing.

Proposition 2.13 Assume $\nu_{+} \in L_{2}(0,1)$ but $\nu_{+} \notin L_{\infty}(0,1)$. Then the semigroup $S^{\alpha, \beta}$ generated by the self-adjoint extension $H_{\alpha, \beta}$ of $H_{+}$is positive.

Proof If $\alpha=0$ then the semigroup is submarkovian by Theorem 2.10. Therefore it remains to prove positivity for $\alpha \neq 0$. Let $P_{\gamma}$ denote the one-dimensional orthogonal projection on $L_{2}(0, \infty)$ with range $\eta_{\gamma}$. Since $\eta_{\gamma}$ is positive (non-negative) the projection $P_{\gamma}$ is a positive operator, i.e. it maps positive functions into positive functions.

Next choose $\gamma$ such that $H_{\alpha, \beta} \geq \gamma I>0$. Since $H_{0,1}=H_{F} \geq H_{\alpha, \beta}$ one also has $H_{0,1}+\gamma I>0$. Then by Krein's theory of lower semibounded extensions there is a $\kappa(\gamma) \geq 0$ such that

$$
\left(\gamma I+H_{\alpha, \beta}\right)^{-1}=\left(\gamma I+H_{0,1}\right)^{-1}+\kappa(\gamma) P_{\gamma}
$$


(see Far75, Theorem 15.1). But $H_{0,1}$ is a submarkovian extension so $\left(\gamma I+H_{0,1}\right)^{-1}$ is a positive operator. In addition $P_{\gamma}$ is a positive operator and $\kappa(\gamma) \geq 0$. It follows immediately that $\left(\gamma I+H_{\alpha, \beta}\right)^{-1}$ is positive for all large $\gamma$. Then $S^{\alpha, \beta}$ is positive by the Trotter product formula.

Proof of Theorem 2.7,II] This follows from Theorem 2.10 and Proposition 2.13,

Remark 2.14 The representation (16) gives information about the possible extension of the resolvents $\left(\gamma I+H_{\alpha, \beta}\right)^{-1}$ to the $L_{p^{-}}$-spaces. Since $H_{0,1}$ is a submarkovian generator it follows from (16) that $\left(\gamma I+H_{\alpha, \beta}\right)^{-1}$ extends to a bounded operator on $L_{p}(0, \infty)$, with $p \in\langle 2, \infty\rangle$, if and only if $P_{\gamma}$ extends to a bounded operator. Now $\eta_{\gamma} \in L_{2}(0, \infty)$ by definition. Therefore $\eta_{\gamma} \in L_{q}(0,1)$ for all $q \in[1,2]$. But since $c$ is bounded away from zero on $[1, \infty\rangle$ it follows that $\eta_{\gamma} \in L_{r}(1, \infty)$ for all $r \in[1, \infty\rangle$ by standard strong ellipticity estimates. Therefore $\eta_{\gamma} \in L_{q}(0, \infty)$ for all $q \in[1,2]$. Hence one concludes that $\left(\gamma I+H_{\alpha, \beta}\right)^{-1}$ extends to a bounded operator on $L_{p}(0, \infty)$ if and only if $\eta_{\gamma} \in L_{p}(0,1)$.

The small $x$ behaviour of $\eta_{\gamma}$ can, however, be deduced from integration of the deficiency equation $\left(\gamma I+H^{*}\right) \eta_{\gamma}=0$. Let $x_{0} \in\langle 0,1]$. After two integrations one finds

$$
\eta_{\gamma}(x)=\eta_{\gamma}\left(x_{0}\right)-\left(c \eta_{\gamma}^{\prime}\right)\left(x_{0}\right) \int_{x}^{x_{0}} d s c(s)^{-1}+\gamma \int_{x}^{x_{0}} d s c(s)^{-1} \int_{s}^{x_{0}} d t \eta_{\gamma}(t)
$$

and this leads to the estimate

$$
\left|\eta_{\gamma}(x)-\eta_{\gamma}\left(x_{0}\right)+\left(c \eta_{\gamma}^{\prime}\right)\left(x_{0}\right) \nu_{x_{0}}(x)\right| \leq \gamma\left\|\eta_{\gamma}\right\|_{2}\left|x-x_{0}\right|^{1 / 2} \nu_{x_{0}}(x)
$$

where $\nu_{x_{0}}(x)=\int_{x}^{x_{0}} d s c(s)^{-1}$. It follows that $\eta_{\gamma} \in L_{p}(0,1)$ if and only if $\nu_{+} \in L_{p}(0,1)$. Therefore $\left(\gamma I+H_{\alpha, \beta}\right)^{-1}$ extends to a bounded operator on $L_{p}(0, \infty)$ with $p \in\langle 2, \infty\rangle$ if and only if $\nu_{+} \in L_{p}(0,1)$.

The question whether the extension of the resolvent to $L_{p}$ is the resolvent of the generator of an $L_{p}$-continuous semigroup seems more complicated.

It remains to prove Theorem [2.7][II], The assumption $\nu_{+} \in L_{\infty}(0,1)$ corresponds to integrability of $c^{-1}$ at the origin. Therefore it is natural to reparametrize and replace $\nu_{+}$ by $\hat{\nu}$ where

$$
\hat{\nu}(x)=\nu_{+}(0)-\nu_{+}(x)=\int_{0}^{x} c^{-1}
$$

and then to replace $\tau_{+}=\nu_{+} \sigma_{+}$by $\hat{\tau}=\hat{\nu} \sigma_{+}$. Since $\hat{\tau}=\nu_{+}(0) \sigma_{+}-\tau_{+}$these replacements make no essential difference to the characterization of $D\left(H_{+}^{*}\right)$ given by Proposition 2.8. Now one has $D\left(H_{+}^{*}\right)=D\left(\bar{H}_{+}\right)+\operatorname{span} \sigma_{+}+\operatorname{span} \hat{\tau}$. The advantage of the reparametrization is that if $\Phi=\varphi+a \sigma_{+}+b \hat{\tau} \in D\left(H_{+}^{*}\right)$ with $\varphi \in D\left(\bar{H}_{+}\right)$then $a=\Phi\left(0_{+}\right)$and $b=\left(c \Phi^{\prime}\right)\left(0_{+}\right)$. This follows since $\varphi\left(0_{+}\right)=0=\left(c \varphi^{\prime}\right)\left(0_{+}\right)$, by Proposition [2.1][II], and $\sigma\left(0_{+}\right)=1,\left(c \sigma_{+}^{\prime}\right)\left(0_{+}\right)=0$, $\hat{\tau}(0)=0$ and $\left(c \hat{\tau}^{\prime}\right)\left(0_{+}\right)=1$ by definition. Now we modify accordingly the definition of the self-adjoint extensions of $H_{+}$.

Let $(\alpha, \beta) \in \mathbf{R}^{2} \backslash(0,0)$. Define

$$
\widehat{D}_{\alpha, \beta}=D\left(\bar{H}_{+}\right)+\operatorname{span}\left(\beta \sigma_{+}+\alpha \hat{\tau}\right) .
$$

Then define the self-adjoint extension $\widehat{H}_{\alpha, \beta}$ of $H$ as the restriction of $H_{+}^{*}$ to $\widehat{D}_{\alpha, \beta}$. Again one has $\widehat{H}_{\alpha, \beta}=\widehat{H}_{\alpha^{\prime}, \beta^{\prime}}$ if and only if $\alpha \beta^{\prime}=\beta \alpha^{\prime}$. Further let $\hat{h}_{\alpha, \beta}$ denote the quadratic form corresponding to $\widehat{H}_{\alpha, \beta}$.

One can again compute the Friedrichs extension of $H_{+}$. 
Proposition 2.15 Assume $\nu_{+} \in L_{\infty}(0,1)$. Then $\bar{h}_{+}=\hat{h}_{1,0}$, the Friedrichs extension $H_{F}$ of $H_{+}$is equal to $\widehat{H}_{1,0}$ and

$$
D\left(H_{F}\right)=\left\{\varphi \in D\left(H_{+}^{*}\right): \varphi\left(0_{+}\right)=0\right\} .
$$

Proof First one has $\hat{h}_{1,0} \supseteq \bar{h}_{+}$and consequently $D\left(\hat{h}_{1,0}\right) \supseteq D\left(\bar{h}_{+}\right)$. Secondly, $\hat{\tau} \in$ $D\left(\bar{h}_{+}\right)$. This follows by standard approximation techniques since $\hat{\tau}$ has compact support, is absolutely continuous and $\hat{\tau}\left(0_{+}\right)=0$. But then $D\left(\widehat{H}_{1,0}\right)=D\left(\bar{H}_{+}\right)+\operatorname{span} \hat{\tau} \subseteq D\left(\bar{h}_{+}\right)$. Since $D\left(\widehat{H}_{1,0}\right)$ is a core of $\hat{h}_{1,0}$ it follows that $D\left(\bar{h}_{+}\right)$is also a core of $\hat{h}_{1,0}$. Therefore $\hat{h}_{1,0}=\bar{h}_{+}$and $\widehat{H}_{1,0}=H_{F}$.

The boundary condition follows since if $\Phi=\varphi+a \sigma_{+}+b \hat{\tau} \in D\left(H_{+}^{*}\right)$ with $\varphi \in D\left(\bar{H}_{+}\right)$ then $\Phi \in D\left(\widehat{H}_{1,0}\right)$ if and only if $a=0$. But it follows from the reparametrization chosen above that $a=\Phi\left(0_{+}\right)$.

The analysis of the remaining self-adjoint extensions of $H_{+}$is in terms of the corresponding quadratic forms. First we observe that there is a unique form domain.

Proposition 2.16 If $\beta \neq 0$ then $D\left(\hat{h}_{\alpha, \beta}\right)=D\left(\bar{h}_{+}\right)+\operatorname{span} \sigma_{+}=D\left(\hat{h}_{0,1}\right)$.

Proof First, since $\hat{h}_{\alpha, \beta} \supseteq \bar{h}_{+}$one has $D\left(\bar{h}_{+}\right) \subseteq D\left(\hat{h}_{\alpha, \beta}\right)$. But $\hat{\tau} \in D\left(\bar{h}_{+}\right)$, as observed in the proof of Proposition 2.15. Moreover, since $D\left(\widehat{H}_{\alpha, \beta}\right) \subseteq D\left(\hat{h}_{\alpha, \beta}\right)$ it follows that $a \sigma_{+}+$ $b \hat{\tau} \in D\left(\hat{h}_{\alpha, \beta}\right)$ for all $a, b$ satisfying $a \alpha=b \beta$. Since $\beta \neq 0$ one deduces that $\sigma_{+} \in D\left(\hat{h}_{\alpha, \beta}\right)$. Therefore $D\left(\hat{h}_{\alpha, \beta}\right) \supseteq D\left(\bar{h}_{+}\right)+\operatorname{span} \sigma_{+}$.

Secondly, we establish the converse inclusion. The proof begins by observing that $D\left(\widehat{H}_{\alpha, \beta}\right)$ is a core of $\hat{h}_{\alpha, \beta}$. Thus if $\Phi \in D\left(\hat{h}_{\alpha, \beta}\right)$ there is a sequence $\Phi_{n} \in D\left(\widehat{H}_{\alpha, \beta}\right)$ which converges to $\Phi$ in the $D\left(\hat{h}_{\alpha, \beta}\right)$-graph norm. But $\Phi_{n}=\varphi_{n}+a_{n} \sigma_{+}+b_{n} \hat{\tau}$ with $\varphi_{n} \in D\left(\bar{H}_{+}\right)$ and $a_{n}, b_{n} \in \mathbf{R}$ satisfying $a_{n} \alpha=b_{n} \beta$. Moreover, $a_{n}=\Phi_{n}\left(0_{+}\right)$and $b_{n}=\left(c \Phi_{n}^{\prime}\right)\left(0_{+}\right)$by the new choice of parameters. Therefore

$\left|a_{n}-a_{m}\right|=\left|\Phi_{n}\left(0_{+}\right)-\Phi_{m}\left(0_{+}\right)\right|=\left|B_{+}\left(\Phi_{n}-\Phi_{m}, \hat{\tau}\right)\right|=\left|\left(H_{+}^{*}\left(\Phi_{n}-\Phi_{m}\right), \hat{\tau}\right)-\left(\left(\Phi_{n}-\Phi_{m}\right), H_{+}^{*} \hat{\tau}\right)\right|$

where the second equality follows because $\hat{\tau}\left(0_{+}\right)=0$ and $\left(c \hat{\tau}^{\prime}\right)\left(0_{+}\right)=1$. But $\left(H^{*} \hat{\tau}\right)(x)$ is bounded with support in the interval [1,2]. Hence $\left|\left(\left(\Phi_{n}-\Phi_{m}\right), H^{*} \hat{\tau}\right)\right| \leq \kappa\left\|\Phi_{n}-\Phi_{m}\right\|_{2}$ for some $\kappa>0$. Moreover, $\hat{\tau} \in D\left(\bar{h}_{+}\right) \subseteq D\left(\hat{h}_{\alpha, \beta}\right)$ by the proof of Proposition 2.15. Hence

$$
\left|\left(H_{+}^{*}\left(\Phi_{n}-\Phi_{m}\right), \hat{\tau}\right)\right|=\left|\left(\widehat{H}_{\alpha, \beta}\left(\Phi_{n}-\Phi_{m}\right), \hat{\tau}\right)\right| \leq \hat{h}_{\alpha, \beta}\left(\Phi_{n}-\Phi_{m}\right)^{1 / 2} \hat{h}_{\alpha, \beta}(\hat{\tau})^{1 / 2} .
$$

Therefore

$$
\left|a_{n}-a_{m}\right| \leq \hat{h}_{\alpha, \beta}\left(\Phi_{n}-\Phi_{m}\right)^{1 / 2} \hat{h}_{\alpha, \beta}(\tau)^{1 / 2}+\kappa\left\|\Phi_{n}-\Phi_{m}\right\|_{2}
$$

and the $a_{n}$ must converge to a limit $a$. Similarly,

$$
\left|b_{n}-b_{m}\right|=\left|\left(c \Phi_{n}^{\prime}\right)\left(0_{+}\right)-\left(c \Phi_{m}^{\prime}\right)\left(0_{+}\right)\right|=\left|B_{+}\left(\Phi_{n}-\Phi_{m}, \sigma_{+}\right)\right|
$$

because $\sigma_{+}\left(0_{+}\right)=1$ and $\left(c \sigma_{+}^{\prime}\right)\left(0_{+}\right)=0$. But then

$$
\left|b_{n}-b_{m}\right| \leq \hat{h}_{\alpha, \beta}\left(\Phi_{n}-\Phi_{m}\right)^{1 / 2} \hat{h}_{\alpha, \beta}\left(\sigma_{+}\right)^{1 / 2}+\kappa\left\|\Phi_{n}-\Phi_{m}\right\|_{2}
$$

and the $b_{n}$ must converge to a limit $b$. One automatically has $a \alpha=b \beta$. 
Next it follows that $\varphi_{n}=\Phi_{n}-a_{n} \sigma_{+}-b_{n} \hat{\tau}$ is $L_{2}$-convergent to a limit $\varphi$. But

$$
\begin{aligned}
\bar{h}_{+}\left(\varphi_{n}-\varphi_{m}\right) & =\hat{h}_{\alpha, \beta}\left(\varphi_{n}-\varphi_{m}\right) \\
& \leq 2 \hat{h}_{\alpha, \beta}\left(\Phi_{n}-\Phi_{m}\right)+4\left|a_{n}-a_{m}\right| \hat{h}_{\alpha, \beta}\left(\sigma_{+}\right)+4\left|b_{n}-b_{m}\right| \hat{h}_{\alpha, \beta}(\hat{\tau})
\end{aligned}
$$

so the $\varphi_{n}$ are convergent in the $D\left(\bar{h}_{+}\right)$-graph norm and one has $\varphi \in D\left(\bar{h}_{+}\right)$. Therefore $\Phi=\varphi+a \sigma_{+}+b \hat{\tau} \in D\left(\bar{h}_{+}\right)+\operatorname{span} \sigma_{+}$. Hence $D\left(\hat{h}_{\alpha, \beta}\right) \subseteq D\left(\bar{h}_{+}\right)+\operatorname{span} \sigma_{+}$.

Finally one can express the forms $\hat{h}_{\alpha, \beta}$ in terms of $\hat{h}_{0,1}$ on their common domain in a classical manner.

Proposition 2.17 If $\nu_{+} \in L_{\infty}(0,1)$ then $D\left(\hat{h}_{\alpha, \beta}\right)=D\left(\hat{h}_{0,1}\right)$ for all $\beta \in \mathbf{R} \backslash\{0\}$ and

$$
\hat{h}_{\alpha, \beta}(\varphi)=\hat{h}_{0,1}(\varphi)+\alpha \beta^{-1}\left|\varphi\left(0_{+}\right)\right|^{2}
$$

for all $\varphi \in D\left(h_{0,1}\right)$.

The self-adjoint extension $\widehat{H}_{\alpha, \beta}$ is the restriction of $H_{+}^{*}$ to the domain

$$
D\left(\widehat{H}_{\alpha, \beta}\right)=\left\{\varphi \in D\left(H_{+}^{*}\right): \beta\left(c \varphi^{\prime}\right)\left(0_{+}\right)=\alpha \varphi\left(0_{+}\right)\right\}
$$

The operators $\widehat{H}_{\alpha, \beta}$ generate positive semigroups on $L_{2}(0, \infty)$ which are submarkovian if and only if $\alpha \beta^{-1} \geq 0$.

Proof The identity of the domains is established in Proposition 2.16. Next we establish the relation between the forms.

First suppose $\Phi=\varphi+a \sigma_{+}+b \hat{\tau}$ with $\varphi \in D\left(\bar{H}_{+}\right)$. Then $\Phi_{1}=\varphi+a \sigma_{+} \in D\left(\widehat{H}_{0,1}\right) \subseteq$ $D\left(\hat{h}_{0,1}\right)$. Moreover, $\hat{\tau} \in D\left(\bar{h}_{+}\right)$as observed in the proof of Proposition 2.15. Therefore $\Phi \in D\left(\hat{h}_{0,1}\right)$. Then one calculates that

$$
\begin{aligned}
\left(\Phi, H_{+}^{*} \Phi\right) & =\hat{h}_{0,1}\left(\Phi_{1}\right)+b\left(\hat{\tau}, H_{+}^{*} \Phi_{1}\right)+b\left(\Phi_{1}, H_{+}^{*} \hat{\tau}\right)+b^{2}\left(\hat{\tau}, H_{+}^{*} \hat{\tau}\right) \\
& =\hat{h}_{0,1}\left(\Phi_{1}\right)+b\left(\hat{\tau}, H_{+}^{*} \Phi_{1}\right)+b\left(H_{+}^{*} \Phi_{1}, \hat{\tau}\right)+b^{2}\left(\hat{\tau}, H_{+}^{*} \hat{\tau}\right)+b B_{+}\left(\Phi_{1}, \hat{\tau}\right) .
\end{aligned}
$$

But

$$
\left(\hat{\tau}, H_{+}^{*} \Phi_{1}\right)=\left(\hat{\tau}, \widehat{H}_{0,1} \Phi_{1}\right)=\hat{h}_{0,1}\left(\hat{\tau}, \Phi_{1}\right)
$$

since $\hat{\tau} \in D\left(\hat{h}_{0,1}\right)$. Similarly $\left(H_{+}^{*} \Phi_{1}, \hat{\tau}\right)=\hat{h}_{0,1}\left(\Phi_{1}, \hat{\tau}\right)$ and $\left(\hat{\tau}, H_{+}^{*} \hat{\tau}\right)=\hat{h}_{0,1}(\hat{\tau})$. Combining these identities gives

$$
\begin{aligned}
\left(\Phi, H_{+}^{*} \Phi\right) & =\hat{h}_{0,1}\left(\Phi_{1}\right)+b \hat{h}_{0,1}\left(\hat{\tau}, \Phi_{1}\right)+b \hat{h}_{0,1}\left(\Phi_{1}, \hat{\tau}\right)+b^{2} \hat{h}_{0,1}(\hat{\tau})+b B_{+}\left(\Phi_{1}, \hat{\tau}\right) \\
& =\hat{h}_{0,1}(\Phi)+b B_{+}\left(\Phi_{1}, \hat{\tau}\right)=\hat{h}_{0,1}(\Phi)+a b
\end{aligned}
$$

where the last step uses the identification $B_{+}\left(\Phi_{1}, \hat{\tau}\right)=a B\left(\sigma_{+}, \hat{\tau}\right)=a$. If, however, one places the restriction $a \alpha=b \beta$ on $a$ and $b$ then $\Phi \in D\left(\widehat{H}_{\alpha, \beta}\right)$ and $H_{+}^{*} \Phi=\widehat{H}_{\alpha, \beta} \Phi$. Therefore

$$
\hat{h}_{\alpha, \beta}(\Phi)=\hat{h}_{0,1}(\Phi)+a b=\hat{h}_{0,1}(\Phi)+\alpha \beta^{-1} a^{2}
$$

for all $\Phi \in D\left(\widehat{H}_{\alpha, \beta}\right)$ and then by closure for all $\Phi \in D\left(\hat{h}_{\alpha, \beta}\right)$. But $a=\Phi\left(0_{+}\right)$so this etablishes the relation (17). 
The boundary condition for $\Phi \in D\left(\widehat{H}_{\alpha, \beta}\right)$ is given by $a \alpha=b \beta$ but the parametrization was chosen such that $a=\Phi\left(0_{+}\right)$and $b=\left(c \Phi^{\prime}\right)\left(0_{+}\right)$.

Finally if $\Phi=\varphi+a \sigma_{+}$with $\varphi \in D\left(\bar{H}_{+}\right)$then since $\left(c \Phi^{\prime}\right)\left(0_{+}\right)=0$ one computes that

$$
\hat{h}_{0,1}(\Phi)=\left(\Phi, H_{+}^{*} \Phi\right)=-\left(\Phi,\left(c \Phi^{\prime}\right)^{\prime}\right)=\int_{0}^{\infty} d x c(x)\left|\Phi^{\prime}(x)\right|^{2} .
$$

Therefore

$$
\hat{h}_{\alpha, \beta}(\Phi)=\int_{0}^{\infty} d x c(x)\left|\Phi^{\prime}(x)\right|^{2}+\left(\alpha \beta^{-1}\right)\left|\Phi\left(0_{+}\right)\right|^{2}
$$

and the positivity and submarkovian properties follow immediately by application of the well known Beurling-Deny criteria (see, for example, [RS78, pages 209-212).

Proof of Theorem 2.7,III This follows directly from Proposition 2.15] and Theorem 2.17. These latter results give a complete description of the self-adjoint extensions of the operator $H$ for $\nu_{+} \in L_{\infty}(0,1)$. The Friedrichs extension $\widehat{H}_{1,0}$ corresponds to Dirichlet boundary conditions $\varphi\left(0_{+}\right)=0$, the extension $\widehat{H}_{0,1}$ to Neumann boundary conditions $\left(c \varphi^{\prime}\right)\left(0_{+}\right)=0$ and the other extensions to Robin boundary conditions. The latter are positive-definite if $\alpha \beta^{-1} \geq 0$.

Remark 2.18 It is straightforward to establish that if $\alpha \beta^{-1}<0$ then the extensions $H_{\alpha, \beta}$ of $H$ have a simple negative eigenvalue. Therefore these extensions are no longer contractive on $L_{2}(0, \infty)$ and certainly not submarkovian.

Remark 2.19 Theorem 2.7 identifies two distinct cases in which $H_{+}$has a unique submarkovian extension, the Friedrichs extension $H_{+F}$. In both cases $\nu_{+} \notin L_{\infty}(0,1)$. Moreover, in both cases $D\left(H_{+F}\right)=D\left(\bar{H}_{+}\right)+\operatorname{span} \sigma_{+}$and $H_{+F}\left(\varphi+a \sigma_{+}\right)=\bar{H}_{+} \varphi-a\left(c \sigma_{+}^{\prime}\right)^{\prime}$ for all $\varphi \in D\left(\bar{H}_{+}\right)$and $a \in \mathbf{R}$. The distinction between the cases occurs because $\nu_{+} \notin L_{2}(0,1)$ implies that $\sigma_{+} \in D\left(\bar{H}_{+}\right)$. Therefore $H_{+F}=\bar{H}_{+}$and $H_{+}$is essentially self-adjoint. But if $\nu_{+} \in L_{2}(0,1)$ then $\sigma_{+} \notin D\left(\bar{H}_{+}\right)$and $H_{+F}$ is a strict extension of $\bar{H}_{+}$.

\section{The line}

In this section we prove Theorem 1.1 by applying the results of Section 2 to $H_{+}=\left.H\right|_{C_{c}^{\infty}(0, \infty)}$ on $L_{2}(0, \infty), H_{-}=\left.H\right|_{C_{c}^{\infty}(-\infty, 0)}$ on $L_{2}(-\infty, 0)$ and $H_{0}=H_{-} \oplus H_{+}$on $L_{2}(\mathbf{R})$.

Let $\sigma \in C_{c}^{\infty}(\mathbf{R})$ satisfy $0 \leq \sigma \leq 1, \operatorname{supp} \sigma \subseteq[-2,2]$ and $\sigma=1$ on $\langle-1,1\rangle$. Define $\sigma_{+}$by $\sigma_{+}(x)=0$ if $x<0$ and $\sigma_{+}(x)=\sigma(x)$ if $x \geq 0$ and set $\sigma_{-}=\sigma-\sigma_{+}$. Further define $\tau_{ \pm}$by $\tau_{ \pm}(x)=\nu_{ \pm}( \pm x) \sigma_{ \pm}(x)$. Thus $\tau_{+}$has support in $[0,2]$ and $\tau_{-}$has support in $[-2,0]$. Now one can characterize the self-adjoint extensions of $H_{+}$with the aid of the functions $\sigma_{+}, \tau_{+}$ exactly as in Section 2 and the extensions of $H_{-}$with the aid of $\sigma_{-}, \tau_{-}$in an analogous fashion.

Proof of Theorem [1.1,I] There are two cases to be considered: $1 . \nu_{+} \wedge \nu_{-} \notin L_{2}(0,1)$, and 2. $\nu_{+} \wedge \nu_{-} \in L_{2}(0,1)$ and $\nu_{+} \vee \nu_{-} \notin L_{2}(0,1)$.

Assume $\nu_{+} \wedge \nu_{-} \notin L_{2}(0,1)$. Then $H_{+}$is essentially self-adjoint on $L_{2}(0, \infty)$ and $H_{-}$ is essentially self-adjoint on $L_{2}(-\infty, 0)$ by Theorem 2.7], Hence $\bar{H}_{0}=\bar{H}_{-} \oplus \bar{H}_{+}$is selfadjoint on $L_{2}(\mathbf{R})$. Since a self-adjoint operator cannot have a proper closed symmetric extension it follows that $\bar{H}=\bar{H}_{0}=\bar{H}_{-} \oplus \bar{H}_{+}$is self-adjoint. Therefore $\bar{H}$ must coincide 
with the Friedrichs extension $H_{F}$ of $H$ and it automatically generates a submarkovian semigroup. Clearly this semigroup must leave $L_{2}(-\infty, 0)$ and $L_{2}(0, \infty)$ invariant.

Secondly, assume $\nu_{+} \notin L_{2}(0,1)$ and $\nu_{-} \in L_{2}(0,1)$. Then the argument is slightly different although the conclusion is the same. Again $H_{+}$is essentially self-adjoint on $L_{2}(0, \infty)$. But then $\sigma_{+} \in D\left(H_{+}^{*}\right)=D\left(\bar{H}_{+}\right)$or, as a relation on $L_{2}(\mathbf{R}), \sigma_{+} \in D(\bar{H})$. Since $\sigma \in D(H)$ it follows that $\sigma_{-} \in D(\bar{H})$. Now one can define a self-adjoint extension $\widetilde{H}_{-}$ of $H_{-}$by $D\left(\widetilde{H}_{-}\right)=D\left(\bar{H}_{-}\right)+\operatorname{span} \sigma_{-} \subseteq D(\bar{H})$ and $\widetilde{H}_{-}\left(\varphi+\beta \sigma_{-}\right)=\bar{H}_{-} \varphi-\beta\left(c \sigma_{-}^{\prime}\right)^{\prime}$ for $\varphi \in D\left(\bar{H}_{-}\right)$and $\beta \in \mathbf{R}$. Then $\bar{H} \supseteq \widetilde{H}_{-} \oplus \bar{H}_{+}$and since a self-adjoint operator cannot have a proper closed symmetric extension it follows that $\bar{H}=\widetilde{H}_{-} \oplus \bar{H}_{+}$is self-adjoint. Then $\bar{H}$ must coincide with the Friedrichs extension $H_{F}$ of $H$ and the corresponding semigroup is submarkovian. Clearly the semigroup leaves $L_{2}(-\infty, 0)$ and $L_{2}(0, \infty)$ invariant.

Thirdly, the argument for $\nu_{+} \in L_{2}(0,1)$ and $\nu_{-} \notin L_{2}(0,1)$ is similar.

Proof of Theorem 1.1,II Now we assume $\nu_{+} \vee \nu_{-} \in L_{2}(0,1)$. Since $H_{0} \subseteq H$ and $H_{0}$ and $H$ are both symmetric one has

$$
H_{0} \subseteq H \subseteq H^{*} \subseteq H_{0}^{*}
$$

But $H_{0}$ is the direct sum of $H_{ \pm}$and as both these operators have deficiency indices $(1,1)$, by Proposition 2.1][I], the operator $H_{0}$ must have deficiency indices $(2,2)$. Thus $D\left(\bar{H}_{0}\right)$ has codimension 4 in $D\left(H_{0}^{*}\right)$. Moreover, $H_{0}^{*}=H_{-}^{*} \oplus H_{+}^{*}$ and one can compute the adjoint of $H_{0}$ in terms of the adjoints of the operators $H_{ \pm}$on the half-lines. But this allows one to compute the domain of $H^{*}$.

Proposition 3.1 If $\nu_{+} \vee \nu_{-} \in L_{2}(0,1)$ then

$$
\begin{aligned}
D\left(H^{*}\right) & =D\left(\bar{H}_{0}\right)+\operatorname{span} \sigma_{+}+\operatorname{span} \sigma_{-}+\operatorname{span}\left(\tau_{+}-\tau_{-}\right) \\
& =D(\bar{H})+\operatorname{span}\left(\sigma_{+}-\sigma_{-}\right)+\operatorname{span}\left(\tau_{+}-\tau_{-}\right) .
\end{aligned}
$$

Proof It follows from Proposition 2.8 applied to $H_{ \pm}$that

$$
D\left(H_{0}^{*}\right)=D\left(\bar{H}_{0}\right)+\operatorname{span} \sigma_{+}+\operatorname{span} \sigma_{-}+\operatorname{span} \tau_{+}+\operatorname{span} \tau_{-} .
$$

Now introduce the boundary form $\varphi, \psi \in D\left(H_{0}^{*}\right) \mapsto B_{0}(\varphi, \psi)$ by

$$
B_{0}(\varphi, \psi)=\left(H_{0}^{*} \varphi, \psi\right)-\left(\varphi, H_{0}^{*} \psi\right) \text {. }
$$

Then it follows from (18) that

$$
D\left(H^{*}\right)=\left\{\varphi \in D\left(H_{0}^{*}\right): B_{0}(\varphi, \psi)=0 \text { for all } \psi \in D(H)\right\}
$$

(see [Far75], lemma on page 86). Now one can compute $D\left(H^{*}\right)$ by use of (20).

First $B_{0}(\varphi, \psi)=0$ for all $\varphi \in D\left(\bar{H}_{0}\right)$ and $\psi \in D(H)$. Secondly

$$
B_{0}\left(\sigma_{+}, \psi\right)=0=B_{0}\left(\sigma_{-}, \psi\right)
$$

for all $\psi \in C_{c}^{\infty}(\mathbf{R})$ by direct calculation. Thirdly,

$$
B_{0}\left(\tau_{+}, \psi\right)=\left(c \nu_{+}^{\prime} \psi\right)(0)-\left(\nu_{+} c \psi^{\prime}\right)(0)=-\psi(0)=B_{0}\left(\tau_{-}, \psi\right)
$$


since $\left(\nu_{+} c \psi^{\prime}\right)(0)=0=\left(\nu_{-} c \psi^{\prime}\right)(0)$ for all $\psi \in C_{c}^{\infty}(\mathbf{R})$. The latter relations follow because the assumption $c(0)=0$ implies that $\left(c \psi^{\prime}\right)(0)=0$. Hence $\left(c \psi^{\prime}\right)(x)=\int_{0}^{x} d s\left(c \psi^{\prime}\right)^{\prime}(s)$ and this gives an estimate $\left|\left(c \psi^{\prime}\right)(x)\right| \leq|x|^{1 / 2}\|H \psi\|_{2}$. As the $\nu_{ \pm}$are square integrable near the origin one has $|x|^{1 / 2} \nu_{ \pm}(x) \rightarrow 0$ as $x \rightarrow 0$ by Lemma 2.4. Therefore $B_{0}\left(\tau_{+}-\tau_{-}, \psi\right)=0$ for all $\psi \in D(H)$. But there is a $\psi \in D(H)$ such that $B_{0}\left(\tau_{+}+\tau_{-}, \psi\right) \neq 0$. Consequently one concludes from (21) that

$$
D\left(H^{*}\right)=D\left(\bar{H}_{0}\right)+\operatorname{span} \sigma_{+}+\operatorname{span} \sigma_{-}+\operatorname{span}\left(\tau_{+}-\tau_{-}\right)
$$

which is the first statement of the proposition. But $\sigma_{+}+\sigma_{-}=\sigma \in C_{c}^{\infty}(\mathbf{R}) \subseteq D(\bar{H})$. Hence $D\left(\bar{H}_{0}\right)+\operatorname{span}\left(\sigma_{+}+\sigma_{-}\right) \subseteq D(\bar{H})$. Therefore

$$
\begin{aligned}
D\left(H^{*}\right) & =D\left(\bar{H}_{0}\right)+\operatorname{span}\left(\sigma_{+}+\sigma_{-}\right)+\operatorname{span}\left(\sigma_{+}-\sigma_{-}\right)+\operatorname{span}\left(\tau_{+}-\tau_{-}\right) \\
& \subseteq D(\bar{H})+\operatorname{span}\left(\sigma_{+}-\sigma_{-}\right)+\operatorname{span}\left(\tau_{+}-\tau_{-}\right) \subseteq D\left(H^{*}\right)
\end{aligned}
$$

which gives the second statement of the proposition.

Note that $B_{0}\left(\tau_{+}, \sigma_{+}\right)=1=B_{0}\left(\tau_{-}, \sigma_{-}\right)$. Hence, under the assumptions of the proposition, one cannot have $\sigma_{+}-\sigma_{-}, \tau_{+}-\tau_{-} \in D(\bar{H})$. Therefore $D(\bar{H})$ has codimension 2 in $D\left(H^{*}\right)$, i.e. $H$ has deficiency indices $(1,1)$. Moreover, since $\sigma \in D(H)$ one has

$$
D\left(H^{*}\right)=D(\bar{H})+\operatorname{span} \sigma_{+}+\operatorname{span}\left(\tau_{+}-\tau_{-}\right)=D(\bar{H})+\operatorname{span} \sigma_{-}+\operatorname{span}\left(\tau_{+}-\tau_{-}\right) .
$$

The self-adjoint extensions $H_{\alpha, \beta}$ of $H$ are given for $(\alpha, \beta) \in \mathbf{R}^{2} \backslash(0,0)$ by

$$
D\left(H_{\alpha, \beta}\right)=D(\bar{H})+\operatorname{span}\left(\beta\left(\sigma_{+}-\sigma_{-}\right)-\alpha\left(\tau_{+}-\tau_{-}\right)\right)
$$

and $H_{\alpha, \beta} \Phi=H^{*} \Phi$ for $\Phi \in D\left(H_{\alpha, \beta}\right)$. This definition is the direct analogue of the definition on the half-line given in Proposition 2.9. Again $H_{\alpha, \beta}=H_{\alpha^{\prime}, \beta^{\prime}}$ for all pairs with $\alpha \beta^{\prime}=\alpha^{\prime} \beta$. In terms of the boundary form $\varphi, \psi \in D\left(H^{*}\right) \mapsto B(\varphi, \psi)$ associated with $H$ one has

$$
D\left(H_{\alpha, \beta}\right)=\left\{\varphi \in D\left(H^{*}\right): B\left(\beta\left(\sigma_{+}-\sigma_{-}\right)-\alpha\left(\tau_{+}-\tau_{-}\right), \varphi\right)=0\right\}
$$

Now consider the extension with $\alpha=0$ and $\beta=1$. Then $D\left(H_{0,1}\right)=D(\bar{H})+\operatorname{span}\left(\sigma_{+}-\sigma_{-}\right)$. But if $\nu_{+} \notin L_{\infty}(0,1)$ then $\sigma_{+} \in D(\bar{h})$ by Lemma 2.11 applied to $h$ instead of $h_{+}$. Since $\sigma=\sigma_{+}+\sigma_{-} \in C_{c}^{\infty}(\mathbf{R})$ it follows that $\sigma_{-} \in D(\bar{h})$. Thus $D\left(H_{0,1}\right) \subseteq D(\bar{h})$. Therefore $h_{0,1}=\bar{h}$ and $H_{0,1}$ is the Friedrichs extension $H_{F}$ of $H$. A similar conclusion is valid if $\nu_{-} \notin L_{\infty}(0,1)$.

Therefore if $\nu_{+} \wedge \nu_{-} \notin L_{\infty}(0,1)$ the operator $H_{0,1}$ generates a submarkovian semigroup $S$ which leaves the subspaces $L_{2}(-\infty, 0)$ and $L_{2}(0, \infty)$ invariant. This establishes the first part of Theorem 1.1.II.

Note that if $\Phi=\varphi+a\left(\sigma_{+}-\sigma_{-}\right)-b\left(\tau_{+}-\tau_{-}\right)$with $\varphi \in D(\bar{H})$ then

$$
\left(c \Phi^{\prime}\right)\left(0_{+}\right)=\lim _{x \rightarrow 0_{+}}\left(c \Phi^{\prime}\right)(x)=-b \lim _{x \rightarrow 0_{+}}\left(c \tau_{+}^{\prime}\right)(x)=b
$$

and

$$
\left(c \Phi^{\prime}\right)\left(0_{-}\right)=\lim _{x \rightarrow 0_{-}}\left(c \Phi^{\prime}\right)(x)=b \lim _{x \rightarrow 0_{-}}\left(c \tau_{-}^{\prime}\right)(x)=-b .
$$


Therefore

$$
b=\left(\left(c \Phi^{\prime}\right)\left(0_{+}\right)-\left(c \Phi^{\prime}\right)\left(0_{-}\right)\right) / 2 .
$$

In particular if $\Phi \in D\left(H_{0,1}\right)$ then $b=0$ and the extension is characterized by the boundary condition $\left(c \Phi^{\prime}\right)\left(0_{+}\right)=\left(c \Phi^{\prime}\right)\left(0_{-}\right)$which links the left and right half-lines.

It remains to prove that under the assumption of the second statement of Theorem 1.1 there are no other submarkovian extensions. The key step in the proof is the identification of the corresponding form domains.

Proposition 3.2 Assume $\nu_{+} \vee \nu_{-} \in L_{2}(0,1)$ but $\nu_{+} \vee \nu_{-} \notin L_{\infty}(0,1)$. If $\alpha \neq 0$ then $D\left(h_{\alpha, \beta}\right)=D(\bar{h})+\operatorname{span}\left(\tau_{+}-\tau_{-}\right)=D\left(h_{1,0}\right)$.

Proof The proof is very similar to the proof of Proposition 2.16,

First, since $h_{\alpha, \beta} \supseteq \bar{h}$ one has $D(\bar{h}) \subseteq D\left(h_{\alpha, \beta}\right)$. But $\sigma_{ \pm} \in D(\bar{h})$, again by Lemma 2.11 and the observation that $\sigma=\sigma_{+}+\sigma_{-} \in C_{c}^{\infty}(\mathbf{R})$. Moreover, since $D\left(H_{\alpha, \beta}\right) \subseteq D\left(h_{\alpha, \beta}\right)$ it follows that $a\left(\sigma_{+}-\sigma_{-}\right)-b\left(\tau_{+}-\tau_{-}\right) \in D\left(h_{\alpha, \beta}\right)$ for all $a, b$ satisfying $a \alpha=b \beta$. Since $\alpha \neq 0$ one deduces that $\tau_{+}-\tau_{-} \in D\left(h_{\alpha, \beta}\right)$. Therefore $D(\bar{h})+\operatorname{span}\left(\tau_{+}-\tau_{-}\right) \subseteq D\left(h_{\alpha, \beta}\right)$.

Secondly, the converse inclusion is established by a slight modification of the second part of the proof of Proposition 2.16. It is again dependent on the observation that $D\left(H_{\alpha, \beta}\right)$ is a core of $h_{\alpha, \beta}$. We omit the details.

Corollary 3.3 Assume $\nu_{+} \vee \nu_{-} \in L_{2}(0,1)$ but $\nu_{+} \vee \nu_{-} \notin L_{\infty}(0,1)$. If $\alpha \neq 0$ then the semigroup $S^{\alpha, \beta}$ generated $H_{\alpha, \beta}$ is neither positive nor $L_{\infty}$-contractive.

Proof It is necessary for positivity of $S^{\alpha, \beta}$ that $\varphi \in D\left(h_{\alpha, \beta}\right)$ implies $|\varphi| \in D\left(h_{\alpha, \beta}\right)$. This is a consequence of the first Beurling-Deny criterion (see, for example, [RS78], page 209). But $\tau_{+}-\tau_{-} \in D\left(h_{1,0}\right)=D\left(h_{\alpha, \beta}\right)$ and by definition the $\tau_{ \pm}$are positive with disjoint supports. Therefore $\left|\tau_{+}-\tau_{-}\right|=\tau_{+}+\tau_{-}$. Since $\tau_{ \pm} \notin D\left(h_{1,0}\right)$ the semigroup is not positive.

The failure of $L_{\infty}$-contractivity is established by the argument used for the half-line (see the proof of Theorem 2.10).

This completes the proof of Theorem 1.1[II.

Proof of Theorem 1.1.III Assume $\nu_{+} \vee \nu_{-} \in L_{2}(0,1)$. Then define $\nu$ by

$$
\nu(x)=\int_{0}^{x} d s c(s)^{-1}
$$

and $\tau=\nu \sigma$. It follows readily that $\tau$ is related to the previous functions $\tau_{ \pm}$by a relation

$$
\tau=\gamma \sigma+\delta\left(\sigma_{+}-\sigma_{-}\right)-\left(\tau_{+}-\tau_{-}\right)
$$

with $\gamma, \delta \in \mathbf{R}$ and $\delta \geq 0$. Therefore the self-adjoint extensions $H_{\alpha, \beta}$ of $H$ can now be defined as the restrictions of $H^{*}$ to the domains $D\left(H_{\alpha, \beta}\right)=D(\bar{H})+\operatorname{span}\left(\beta\left(\sigma_{+}-\sigma_{-}\right)+\alpha \tau\right)$ with a typical element $\Phi \in D\left(H_{\alpha, \beta}\right)$ given by $\Phi=\varphi+a\left(\sigma_{+}-\sigma_{-}\right)+b \tau$ where $\varphi \in D(\bar{H})$ and $a \alpha=b \beta$. Therefore $\Phi\left(0_{ \pm}\right)=\varphi(0) \pm a,\left(c \Phi^{\prime}\right)\left(0_{ \pm}\right)= \pm b$ and one has $a=\left(\Phi\left(0_{+}\right)-\Phi\left(0_{-}\right)\right) / 2$ and $b=\left(\left(c \Phi^{\prime}\right)\left(0_{+}\right)-\left(c \Phi^{\prime}\right)\left(0_{-}\right)\right) / 2$. Thus $\Phi$ satisfies the boundary condition

$$
\beta\left(\left(c \Phi^{\prime}\right)\left(0_{+}\right)-\left(c \Phi^{\prime}\right)\left(0_{-}\right)\right)=\alpha\left(\Phi\left(0_{+}\right)-\Phi\left(0_{-}\right)\right) .
$$


A slight variation of the previous arguments gives $h_{F}=h_{1,0}$ and $D\left(H_{F}\right)=D(\bar{H})+$ $\operatorname{span} \tau$. The corresponding boundary condition is $\left(c \Phi^{\prime}\right)\left(0_{+}\right)=\left(c \Phi^{\prime}\right)\left(0_{-}\right)$. Although the Friedrichs extension is automatically submarkovian the corresponding semigroup no longer leaves the subspaces $L_{2}(0, \infty)$ and $L_{2}(-\infty, 0)$ invariant. The boundary condition now links the two sides of the line.

If $\beta \neq 0$ then arguing as in the proofs of Propositions 2.16 and 3.2 one finds

$$
D\left(h_{\alpha, \beta}\right)=D(\bar{h})+\operatorname{span}\left(\sigma_{+}-\sigma_{-}\right)=D\left(h_{0,1}\right)
$$

Then by an argument analogous to the proof of Proposition 2.17 one deduces that

$$
h_{\alpha, \beta}(\varphi)=h_{0,1}(\varphi)+\alpha \beta^{-1} \mid\left(\varphi\left(0_{+}\right)-\left.\varphi\left(0_{-}\right)\right|^{2} / 4\right.
$$

Since $D\left(H_{0,1}\right)$ corresponds to the boundary condition $\left(c \varphi^{\prime}\right)\left(0_{+}\right)=\left(c \varphi^{\prime}\right)\left(0_{-}\right)$it follows that

$$
h_{0,1}(\varphi)=\int_{\mathbf{R}} d x c(x)\left|\varphi^{\prime}(x)\right|^{2}
$$

for all $\varphi \in D\left(H_{0,1}\right)$. Then, by closure, $h_{0,1}$ is a Dirichlet form and $H_{0,1}$ is submarkovian.

Since ||$\varphi(x)|-| \varphi(y)|| \leq|\varphi(x)-\varphi(y)|$ it follows that if $\alpha \beta^{-1} \geq 0$ then $h_{\alpha, \beta}$ satisfies the first Beurling-Deny criterion. A similar argument shows that under the same restriction on $\alpha$ and $\beta$ it satisfies the second criterion. Therefore one concludes that if $\alpha \beta^{-1} \geq 0$ then $H_{\alpha, \beta}$ is submarkovian.

Finally we note that if $\alpha \beta^{-1}<0$ one can establish that $H_{\alpha, \beta}$ has a simple negative eigenvalue. So $S^{\alpha, \beta}$ is not contractive on $L_{2}(\mathbf{R})$ and therefore not submarkovian.

Remark 3.4 If $H$ has a unique submarkovian extension then it is equal to the Friedrichs extension $H_{F}$ and is given by $D\left(H_{F}\right)=D(\bar{H})+\operatorname{span}\left(\sigma_{+}-\sigma_{-}\right)$and

$$
H_{F}\left(\varphi+a\left(\sigma_{+}-\sigma_{-}\right)\right)=\bar{H} \varphi-a\left(\left(c \sigma_{+}^{\prime}\right)^{\prime}-\left(c \sigma_{-}^{\prime}\right)^{\prime}\right)
$$

for all $\varphi \in D(\bar{H})$ and $a \in \mathbf{R}$. There are two distinct cases corresponding to the first two cases of Theorem [1.1. In the first case, $\nu_{+} \vee \nu_{-} \notin L_{2}(0,1)$, one has $\sigma_{ \pm} \in D(\bar{H})$. Therefore $H_{F}=\bar{H}$ and $H$ is essentially self-adjoint. In the second case $\sigma_{ \pm} \notin D(\bar{H})$.

\section{$4 \quad L_{1}$-estimates}

The principal aim of this section is the proof of Theorem 1.2. This requires a number of preliminary $L_{1}$-estimates which are valid under the weaker hypothesis $c \in W_{\text {loc }}^{1,1}(\mathbf{R})$. This is sufficient to ensure that $H C_{c}^{\infty}(\mathbf{R}) \subseteq L_{1}(\mathbf{R})$. We again begin by analyzing $H$ on the half-line.

Let $H_{+}=\left.H\right|_{C_{c}^{\infty}(0, \infty)}$. Then $H_{+} C_{c}^{\infty}(0, \infty) \subseteq L_{1}(0, \infty)$ and we may consider $H_{+}$as an operator on $L_{1}(0, \infty)$ with domain $D\left(H_{+}\right)=C_{c}^{\infty}(0, \infty)$. Next let $\sigma_{+} \in C_{c}^{\infty}(0, \infty)$ be the function defined in Section 2 and define the extension $\widetilde{H}_{+}$of $H_{+}$by setting $D\left(\widetilde{H}_{+}\right)=$ $D\left(H_{+}\right)+\operatorname{span} \sigma_{+}$and $\widetilde{H}_{+}\left(\varphi+\beta \sigma_{+}\right)=H_{+} \varphi-\beta\left(c \sigma_{+}^{\prime}\right)^{\prime}$ for all $\varphi \in D\left(H_{+}\right)$and $\beta \in \mathbf{R}$. An analogous $L_{2}$-extension was used in the proof of Theorem 1.1I.

Lemma 4.1 The operator $\widetilde{H}_{+}$is both $L_{1}$-dissipative and $L_{1}$-dispersive. Therefore $\widetilde{H}_{+}$is $L_{1}$-closable and its closure is $L_{1}$-dissipative and $L_{1}$-dispersive. 
Proof The operator $\widetilde{H}_{+}$is $L_{1}$-dissipative if and only if

$$
\left(\widetilde{H}_{+} \Phi, \operatorname{sgn}(\Phi)\right) \geq 0
$$

for all $\Phi \in D\left(\widetilde{H}_{+}\right)$where $\operatorname{sgn}(\Phi)$ denotes the usual sign function. Moreover, it is $L_{1^{-}}$ dispersive if and only if

$$
\left(\widetilde{H}_{+} \Phi,(\operatorname{sgn}(\Phi) \vee 0)\right) \geq 0
$$

for all $\Phi \in D\left(\widetilde{H}_{+}\right)$. (For background on dissipative and dispersive operators see [BaR84], Section 2.1, or Nag86.)

Let $\Phi=\varphi+\beta \sigma_{+}$with $\varphi \in D\left(H_{+}\right)$and $\beta \in \mathbf{R}$ and note that $\left(c \Phi^{\prime}\right)\left(0_{+}\right)=0$. Next choose a monotonically increasing $C^{\infty}$-function $\eta$ such that $\eta(x)=0$ if $|x| \leq 1$ and $\eta(x)= \pm 1$ if $\pm x \geq 2$. Then set $\Phi_{n}=\eta(n \Phi)$. It follows that $\Phi_{n} \in C_{c}^{\infty}(\mathbf{R})$ and $\Phi_{n}$ converges pointwise to $\operatorname{sgn}(\Phi)$ as $n \rightarrow \infty$. But integrating by parts and using $\left(c \Phi^{\prime}\right)\left(0_{+}\right)=0$ one has

$$
\left(\widetilde{H}_{+} \Phi, \Phi_{n}\right)=\int_{0}^{\infty} d x c(x) \Phi^{\prime}(x) \Phi_{n}^{\prime}(x)=\int_{0}^{\infty} d x c(x)\left|\Phi^{\prime}(x)\right|^{2} n \eta^{\prime}(n \Phi) \geq 0 .
$$

Therefore in the limit $n \rightarrow \infty$ one deduces that $\left(\widetilde{H}_{+} \Phi, \operatorname{sgn}(\Phi)\right) \geq 0$. Thus $\widetilde{H}_{+}$is $L_{1^{-}}$ dissipative. The proof of dispersivity is similar.

Finally it follows by general theory that a norm densely-defined dissipative operator on a Banach space is closable and that its closure is dissipative. Moreover, if the operator is dispersive then the closure is also dispersive. (See [BaR84], Theorem 2.3.1.)

Note that as $H_{+}$is a restriction of $\widetilde{H}_{+}$it automatically inherits the dissipativity and dispersivity properties. Thus $H_{+}$is both dissipative and dispersive on $L_{1}(0, \infty)$. Therefore its $L_{1}$-closure $\bar{H}_{+}^{1}$ generates a strongly continuous positive contraction semigroup on $L_{1}(0, \infty)$ if and only if the range of $\left(I+H_{+}\right)$is $L_{1}$-norm dense (see, BaR84, Corollary 2.2.2). But this is equivalent to the statement that if $\psi \in L_{\infty}(0, \infty)$ and $\left(\psi,\left(I+H_{+}\right) \varphi\right)=0$ for all $\varphi \in C_{c}^{\infty}(0, \infty)$ then $\psi=0$, i.e. if $\left(I+H_{+}^{*}\right) \psi=0$ in the sense of distributions then $\psi=0$. If, however, $\psi-\left(c \psi^{\prime}\right)^{\prime}=0$ in the distributional sense it follows that $c \psi^{\prime}$ is locally absolutely continuous. Then since $c>0$ on $\langle 0, \infty\rangle$ it follows that $\psi$ is a $C^{1}$-function locally. Therefore $\psi=\left(c \psi^{\prime}\right)^{\prime}$ in the usual sense of ordinary differential equations.

The following lemma is the key to establishing the range condition.

Lemma 4.2 Let $c \in W_{\mathrm{loc}}^{1,1}(0, \infty)$ be strictly positive on $\langle 0, \infty\rangle$. Assume $\int_{1}^{\infty} d s s c(s)^{-1}=$ $\infty$. Consider the ordinary differential equation $\left(c \psi^{\prime}\right)^{\prime}=\psi$ on $\langle 0, \infty\rangle$ with the boundary condition $\psi^{\prime}\left(0_{+}\right)=\gamma \psi\left(0_{+}\right)$.

If $\gamma \geq 0$ then there are no non-zero $L_{p}$-solutions $\psi$ for any $p \in[1, \infty]$.

Proof Assume that $\psi$ is a non-zero solution. Then

$$
\begin{aligned}
-\int_{0}^{x}|\psi|^{2}=-\int_{0}^{x}\left(c \psi^{\prime}\right)^{\prime} \psi & =\left(c \psi^{\prime} \psi\right)\left(0_{+}\right)-\left(c \psi^{\prime} \psi\right)(x)+\int_{0}^{x} c\left|\psi^{\prime}\right|^{2} \\
& =\gamma \psi^{2}\left(0_{+}\right)-\left(c \psi^{\prime} \psi\right)(x)+\int_{0}^{x} c\left|\psi^{\prime}\right|^{2}
\end{aligned}
$$

Therefore

$$
2^{-1}\left(c\left(\psi^{2}\right)^{\prime}\right)(x)=\gamma \psi^{2}\left(0_{+}\right)+\int_{0}^{x}|\psi|^{2}+\int_{0}^{x} c\left|\psi^{\prime}\right|^{2} \geq \int_{0}^{x}|\psi|^{2} .
$$


where the last bound uses $\gamma \geq 0$. In particular $\psi^{2}$ is non-decreasing. Since $\psi \neq 0$ there is an $x_{0}$ such that $\psi\left(x_{0}\right) \neq 0$ and it follows that

$$
2^{-1}\left(c\left(\psi^{2}\right)^{\prime}\right)(x) \geq\left(x-x_{0}\right) \psi^{2}\left(x_{0}\right)
$$

for all $x \geq x_{0}$. Therefore

$$
\left(\psi^{2}\right)^{\prime}(x) \geq \psi^{2}\left(x_{0}\right) x c(x)^{-1}
$$

for $x \geq 2 x_{0}$. It follows by integration that $|\psi(x)|^{2} \rightarrow \infty$ as $x \rightarrow \infty$. Therefore there are no non-zero $L_{p}$-solutions.

Remark 4.3 The conclusion of the lemma is valid in the limiting case $\gamma=+\infty$, i.e. with the Dirichlet boundary condition $\psi\left(0_{+}\right)=0$.

If $\psi \in L_{\infty}(0, \infty)$ and $\left(\psi,\left(I+H_{+}\right) \varphi\right)=0$ for all $\varphi \in C_{c}^{\infty}(0, \infty)$ then $\psi$ satisfies the differential equation of Lemma 4.2 but it is not clear that it satisfies an appropriate boundary condition. This will follow from an $L_{1}$-version of Lemma 2.11.

Proposition 4.4 If $\nu_{+} \notin L_{\infty}(0,1)$ then $\sigma_{+} \in D\left(\bar{H}_{+}^{1}\right)$ and $\bar{H}_{+}^{1} \sigma_{+}=-\left(c \sigma_{+}^{\prime}\right)^{\prime} \in L_{1}(0, \infty)$.

Proof First observe that $C_{c}^{2}(0, \infty) \subseteq D\left(\bar{H}_{+}^{1}\right)$ by straightforward estimates. Then let $\varphi_{n}$ be a sequence of $C^{2}$-functions satisfying $0 \leq \varphi_{n} \leq 1, \varphi_{n}(x)=0$ if $x \in\left[0, n^{-1}\right]$ and $\varphi_{n}(x)=1$ if $x \geq 1$. It follows that $0 \leq \varphi_{n} \sigma_{+} \leq 1, \operatorname{supp} \varphi_{n} \sigma_{+} \in\left[n^{-1}, 2\right], \varphi_{n} \sigma_{+} \in D\left(\bar{H}_{+}^{1}\right)$ and $\bar{H}_{+}^{1}\left(\varphi_{n} \sigma_{+}\right)=-\left(c\left(\varphi_{n} \sigma_{+}\right)^{\prime}\right)^{\prime}$.

Secondly, we construct below a particular sequence of $\varphi_{n}$ such that

$$
\lim _{n \rightarrow \infty} \varphi_{n}(x)=1
$$

for all $x>0$ and

$$
\lim _{n \rightarrow \infty}\left\|\left(c \varphi_{n}^{\prime}\right)^{\prime}\right\|_{1}=0
$$

Then it follows that

$$
\lim _{n \rightarrow \infty}\left\|\varphi_{n} \sigma_{+}-\sigma_{+}\right\|_{1}=0 \quad \text { and } \quad \lim _{n \rightarrow \infty}\left\|\bar{H}_{+}^{1}\left(\varphi_{n} \sigma_{+}\right)+\left(c \sigma_{+}^{\prime}\right)^{\prime}\right\|_{1}=0
$$

and the proposition is established.

Construction of the sequence $\varphi_{n}$ The construction is in four steps.

Step 1 Define $\chi_{n}: \mathbf{R} \rightarrow[0,1]$ by (14). Then set $\xi_{n}=\left(1-\chi_{n}\right)^{2}$. The $\xi_{n}$ are positive, increasing, differentiable and $\xi_{n}(x) \rightarrow 1$ for all $x>0$ as $n \rightarrow \infty$. Moreover, on $\left[n^{-1}, 1\right]$ one has $\xi_{n}^{\prime}=-2 \chi_{n}^{\prime}\left(1-\chi_{n}\right)$ and $\xi_{n}^{\prime}=0$ elsewhere. But the definition of $\chi_{n}$ gives

$$
\xi_{n}^{\prime}(x)=2 c(x)^{-1}\left(\int_{n^{-1}}^{x} c^{-1}\right) \nu_{n}^{-2}
$$

for $x \in\left[n^{-1}, 1\right]$. In particular $\xi_{n}^{\prime}\left(n^{-1}\right)=0$ and $\xi_{n}^{\prime}(1)=2 c(1)^{-1} \nu_{n}^{-1}$. Thus $\xi_{n}$ fails to be twice-differentiable since $\xi_{n}^{\prime}$ is discontinuous at $x=1$. Therefore we modify the derivative by the addition of a linear function on the interval $\left[n^{-1}, 1\right]$. 
Step 2 Define $\eta_{n}$ by

$$
\eta_{n}(x)= \begin{cases}0 & \text { if } x \in\left[0, n^{-1}\right\rangle \\ \left(\xi_{n}^{\prime}(x)-\xi_{n}^{\prime}(1)\left(x-n^{-1}\right)\left(1-n^{-1}\right)^{-1}\right) & \text { if } x \in\left[n^{-1}, 1\right] \\ 0 & \text { if } x \geq 1\end{cases}
$$

then $\eta_{n}\left(n^{-1}\right)=0=\eta_{n}(1)$ and $\eta_{n}$ is continuous. Therefore setting $\zeta_{n}(x)=\int_{0}^{x} \eta_{n}$ for $x \leq 1$ and $\zeta_{n}(x)=\zeta_{n}(1)$ if $x \geq 1$ the resulting function is twice-differentiable and $\zeta_{n}(x)=0$ for $x \in[0,1 / n]$. Nevertheless

$$
\zeta_{n}(1)=\int_{0}^{1} \eta_{n}<\xi_{n}(1)=1
$$

so to complete the construction we rescale $\zeta_{n}$.

Step 3 Define $\varphi_{n}=\zeta_{n}(1)^{-1} \zeta_{n}$. It follows immediately that $\varphi_{n}(x)=0$ if $x \in\left[0, n^{-1}\right]$, $\varphi_{n}(x)=1$ if $x \geq 1$ and $\varphi_{n}$ is twice differentiable. Moreover,

$$
\begin{aligned}
\varphi_{n}(x) & =\zeta_{n}(1)^{-1}\left(1-\xi_{n}^{\prime}(1) \int_{n^{-1}}^{x} d s\left(s-n^{-1}\right)\left(1-n^{-1}\right)^{-1}\right) \\
& \geq \zeta_{n}(1)^{-1}\left(1-\xi_{n}^{\prime}(1)\right) \geq\left(1-\xi_{n}^{\prime}(1)\right)
\end{aligned}
$$

and since $\xi_{n}^{\prime}(1)=c(1)^{-1} \nu_{n}^{-1} \rightarrow 0$ as $n \rightarrow \infty$ one has $\varphi_{n} \geq 0$ for all sufficiently large $n$. It remains to verify (22) and (23).

Step 4 First one has $\left.1>\zeta_{n}(1) \geq 1-\xi_{n}^{\prime}(1)\right) \rightarrow 1$ as $n \rightarrow \infty$. Therefore $\lim _{n \rightarrow \infty} \varphi_{n}(x)=$ $\lim _{n \rightarrow \infty} \zeta_{n}(x)$. But

$$
\zeta_{n}(x)=\xi_{n}(x)-\xi_{n}^{\prime}(1) \int_{n^{-1}}^{x} d s\left(s-n^{-1}\right)\left(1-n^{-1}\right)^{-1} \rightarrow 1
$$

for $x>0$ since $\xi_{n}^{\prime}(1) \rightarrow 0$. Thus (22) is verified.

Secondly, if $x \in\left[n^{-1}, 1\right]$ then

$$
\left(c \varphi_{n}^{\prime}\right)(x)=\zeta_{n}(1)^{-1}(c \eta)(x)=\zeta_{n}(1)^{-1}\left(\left(c \xi_{n}^{\prime}\right)(x)-\xi_{n}^{\prime}(1) c(x)\left(x-n^{-1}\right)\left(1-n^{-1}\right)^{-1}\right) .
$$

Therefore

$$
\left(c \varphi_{n}^{\prime}\right)^{\prime}(x)=\zeta_{n}(1)^{-1}\left(\left(c \xi_{n}^{\prime}\right)^{\prime}(x)-\xi_{n}^{\prime}(1) c^{\prime}(x)\left(x-n^{-1}\right)\left(1-n^{-1}\right)^{-1}-\xi_{n}^{\prime}(1) c(x)\left(1-n^{-1}\right)^{-1}\right) .
$$

But $\zeta_{n}(1)^{-1} \rightarrow 1, \xi_{n}^{\prime}(1) \rightarrow 0$ and $\left\|c^{\prime}\right\|_{\infty}<\infty$. Thus

$$
\lim _{n \rightarrow \infty}\left\|\left(c \varphi_{n}^{\prime}\right)^{\prime}\right\|_{1}=\lim _{n \rightarrow \infty}\left\|\left(c \xi_{n}^{\prime}\right)^{\prime}\right\|_{1}
$$

i.e. the modifications to $\xi_{n}$ in Steps 2 and 3 do not affect the $L_{1}$-limit. But

$$
\left(c \xi_{n}^{\prime}\right)^{\prime}(x)=c(x)^{-1} \nu_{n}^{-2}
$$

for $x \in\left[n^{-1}, 1\right]$ and is zero elsewhere. Therefore

$$
\left\|\left(c \xi_{n}^{\prime}\right)^{\prime}\right\|_{1}=\left(\int_{n^{-1}}^{1} c^{-1}\right) \nu_{n}^{-2}=\nu_{n}^{-1}
$$

and (23) is verified.

This completes the proof of Proposition 4.4. 
Corollary 4.5 If $\nu_{+} \notin L_{\infty}(0,1)$ then the $L_{1}$-closures of $H_{+}$and $\widetilde{H}_{+}$are equal.

Proof This follows immediately because $D\left(H_{+}\right) \subseteq D\left(\widetilde{H}_{+}\right)$but the proposition establishes that $D\left(\widetilde{H}_{+}\right) \subseteq D\left(\bar{H}_{+}^{1}\right)$.

Remark 4.6 It follows from the proof of Proposition 4.4 that if $\nu_{+} \notin L_{\infty}(0,1)$ then one may construct a sequence $\sigma_{n} \in C_{c}^{\infty}(0, \infty)$ such that $\left\|\sigma_{n}-\sigma_{+}\right\|_{1} \rightarrow 0$ and moreover $\left\|H_{+} \sigma_{n}+\left(c \sigma_{+}^{\prime}\right)^{\prime}\right\|_{1} \rightarrow 0$ as $n \rightarrow \infty$. It suffices to replace the $C_{c}^{2}$-approximants $\varphi_{n}$ by $C_{c}^{\infty}$-approximants and to set $\sigma_{n}=\varphi_{n} \sigma_{+}$.

Remark 4.7 Although the foregoing results were established for $H_{+}=\left.H\right|_{C_{c}^{\infty}(0, \infty)}$ similar statements are true for $H_{-}=\left.H\right|_{C_{c}^{\infty}(-\infty, 0)}$, e.g. $H_{-}$is $L_{1}$-dissipative on $L_{1}(-\infty, 0)$.

Now we turn to the proof of Theorem 1.2. Since this involves the action of $H$ on $L_{1}$ and on $L_{2}$ it is necessary to adopt the earlier stronger assumption that $c \in W_{\mathrm{loc}}^{1,2}(\mathbf{R})$. Then all the preceding results apply.

Proof of Theorem $1.2 \llbracket \rightrightarrows$ II, This is evident.

II $\Rightarrow$ III. First, it follows from an obvious extension of Lemma 4.1 that $H$ is both $L_{1^{-}}$ dissipative and $L_{1}$-dispersive. Therefore Condition II implies that the $L_{1}$-closure $\bar{H}^{1}$ of $H$ generates a strongly continuous positive contraction semigroup $S$ on $L_{1}(\mathbf{R})$. Next let $H_{F}$ denote the Friedrichs extension of $H$ and $H_{1}$ the generator of the corresponding submarkovian semigroup acting on $L_{1}(\mathbf{R})$. If $\varphi \in C_{c}^{\infty}(\mathbf{R})$ then $\bar{H}^{1} \varphi=H \varphi=H_{F} \varphi$. But $H \varphi \in L_{1}(\mathbf{R})$. Therefore $\varphi \in D\left(H_{1}\right)$ and $H_{1} \varphi=H_{F} \varphi=\bar{H}^{1} \varphi$. Since $C_{c}^{\infty}(\mathbf{R})$ is a core of $\bar{H}^{1}$ it follows that $H_{1} \supseteq \bar{H}^{1}$. But $\bar{H}^{1}$ generates a contraction semigroup and $H_{1}$ is $L_{1}$-dissipative. Therefore $\bar{H}_{1}=\bar{H}^{1}$. In particular $S$ extends to a submarkovian semigroup on the $L_{p}$-spaces which coincides with the submarkovian semigroup generated by $H_{F}$.

Secondly, let $\widetilde{H}$ be another submarkovian extension of $H$ and $\widetilde{H}_{1}$ the generator of the corresponding semigroup on $L_{1}(\mathbf{R})$. If $\varphi \in C_{c}^{\infty}(\mathbf{R})$ one then has $\bar{H}^{1} \varphi=H \varphi=$ $\widetilde{H} \varphi \in L_{1}(\mathbf{R})$. Therefore $\widetilde{H}_{1} \varphi=\widetilde{H} \varphi=\bar{H}^{1} \varphi$ and it follows by the previous argument that $\widetilde{H}_{1}=\bar{H}^{1}$. Hence $\widetilde{H}_{1}=H_{1}$ and $\widetilde{H}$ must be the Friedrichs extension of $H$. Therefore the Friedrichs extension is the unique submarkovian extension of $H$.

III $\Rightarrow$ I. It follows from Theorem 1.2 that $H$ has a unique submarkovian extension, the Friedrichs extension, if and only if $\nu_{+} \vee \nu_{-} \notin L_{\infty}(0,1)$. Let us assume $\nu_{+} \notin L_{\infty}(0,1)$ but $\nu_{-} \in L_{\infty}(0,1)$. The other cases are handled similarly.

First, we argue that $\left(I+H_{+}\right) C_{c}^{\infty}(0, \infty)$ is dense in $L_{1}(0, \infty)$. Let $\psi \in L_{\infty}(0, \infty)$ such that $\left(\psi,\left(I+H_{+}\right) \varphi\right)=0$ for all $\varphi \in C_{c}^{\infty}(0, \infty)$. Then $\psi$ satisfies the ordinary differential equation $\left(c \psi^{\prime}\right)^{\prime}=\psi$. In particular $c \psi^{\prime}$ is continuous near the origin and $\left(c \psi^{\prime}\right)\left(0_{+}\right)$exists. But if $\left(c \psi^{\prime}\right)\left(0_{+}\right) \neq 0$ then $\psi \sim \nu_{+}$as $x \rightarrow 0_{+}$. Since $\nu_{+} \notin L_{\infty}(0,1)$ this contradicts the boundedness of $\psi$. Therefore $\left(c \psi^{\prime}\right)\left(0_{+}\right)=0$. Then the assumed growth conditions at infinity allow the application of Lemma 4.2 and one deduces that $\psi=0$. Therefore $\left(I+H_{+}\right) C_{c}^{\infty}(0, \infty)$ is dense in $L_{1}(0, \infty)$.

Secondly, consider the restriction $H_{-}$of $H$ to $C_{c}^{\infty}(-\infty, 0)$. Since $\nu_{-} \in L_{\infty}(0,1)$ one cannot apply the foregoing reasoning to establish that $\left(I+H_{-}\right) C_{c}^{\infty}(-\infty, 0)$ is dense in $L_{1}(-\infty, 0)$. It follows, however, from Proposition 4.4 that $\sigma_{+} \in D\left(\bar{H}_{+}^{1}\right)$, or $\sigma_{+} \in D\left(\bar{H}^{1}\right)$ on $L_{1}(\mathbf{R})$. Since $\sigma_{+} \in C_{c}^{\infty}(\mathbf{R})=D(H)$ it follows that $\sigma_{-}=\sigma-\sigma_{+} \in D\left(\bar{H}^{1}\right)$. Now one can define a dissipative extension $\widetilde{H}_{-}$of $H_{-}$by $D\left(\widetilde{H}_{-}\right)=D\left(H_{-}\right)+\operatorname{span} \sigma_{-}$and 
$\widetilde{H}_{-}\left(\varphi+\beta \sigma_{-}\right)=H_{-} \varphi-\beta\left(c \sigma_{-}^{\prime}\right)^{\prime}$ for all $\varphi \in D\left(H_{-}\right)$and $\beta \in \mathbf{R}$. Then $\bar{H} \supseteq \widetilde{H}_{-} \oplus H_{+}$and to deduce that $(I+H) C_{c}^{\infty}(\mathbf{R} \backslash\{0\})$ is dense in $L_{1}(\mathbf{R})$ it suffices to prove that $\left(I+\widetilde{H}_{-}\right) D\left(\widetilde{H}_{-}\right)$ is dense in $L_{1}(-\infty, 0)$.

Fourthly, suppose there is a $\psi \in L_{\infty}(-\infty, 0)$ such that $\left(\psi,\left(I+\widetilde{H}_{-}\right) \Phi\right)=0$ for all $\Phi=\varphi+\beta \sigma_{-} \in D\left(\widetilde{H}_{-}\right)$. It follows that

$$
\left(\psi,\left(I+H_{-}\right) \varphi\right)+\beta\left(\left(\psi, \sigma_{-}\right)-\left(\psi,\left(c \sigma_{-}^{\prime}\right)^{\prime}\right)\right)=0
$$

for all $\varphi \in C_{c}^{\infty}(-\infty, 0)$ and all $\beta \in \mathbf{R}$. Therefore $\left(\psi,\left(I+H_{-}\right) \varphi\right)=0$ for all $\varphi \in C_{c}^{\infty}(-\infty, 0)$ as before. In addition, however, one must have

$$
(\psi, \mu)-\left(\psi,\left(c \mu^{\prime}\right)^{\prime}\right)=0
$$

for all $\mu=\sigma_{-}\left(\bmod C_{c}^{\infty}(-\infty, 0)\right)$. But integration by parts gives

$$
(\psi, \mu)=\left(\left(c \psi^{\prime}\right)^{\prime}, \mu\right)-\left(\mu c \psi^{\prime}\right)\left(0_{-}\right)
$$

again for all $\mu=\sigma_{-}\left(\bmod C_{c}^{\infty}(-\infty, 0)\right)$. This immediately implies that $\left(c \psi^{\prime}\right)\left(0_{-}\right)=0$. Finally, arguing as above, there is no non-zero bounded $\psi$ satisfying $\left(\psi,\left(I+H_{-}\right) \varphi\right)=0$ for all $\varphi \in C_{c}^{\infty}(-\infty, 0)$ and $\left(c \psi^{\prime}\right)\left(0_{-}\right)=0$. So $\left(I+\widetilde{H}_{-}\right) D\left(\widetilde{H}_{-}\right)$is dense in $L_{1}(-\infty, 0)$.

Conditions [ and [I] of Theorem 1.2 imply that $C_{c}^{\infty}(\mathbf{R})$ is a core of the generator $H_{1}$ of the submarkovian semigroup $S$ acting on $L_{1}(\mathbf{R})$. This in turn implies that the semigroup is conservative. Indeed one has $(H \varphi, \mathbb{1})=0$ for all $\varphi \in C_{c}^{\infty}(\mathbf{R})$ and by closure for all $\varphi \in D\left(H_{1}\right)$. Then, however,

$$
\frac{d}{d t}\left(S_{t} \varphi, \mathbb{1}\right)=\left(H_{1} S_{t} \varphi, \mathbb{1}\right)=0
$$

$\varphi \in D\left(H_{1}\right)$. Therefore $S$ must be conservative. Davies [Dav85], Theorem 2.2, has established a converse statement for a large class of elliptic operators on $\mathbf{R}^{d}$ (see also [Pan88]).

\section{$5 \quad$ Lipschitz coefficients}

In this section we examine operators with a coefficient $c \in W_{\text {loc }}^{1, \infty}(\mathbf{R})$ and give a proof of Theorem 1.3. The simplest case is for $c$ strictly positive on $\mathbf{R} \backslash\{0\}$ but $c(0)=0$. Then the $W_{\text {loc }}^{1, \infty}$-assumption on $c$ ensures that $c(x)=O(x)$ as $x \rightarrow 0_{ \pm}$. Thus $\nu_{ \pm} \notin L_{\infty}(0,1)$ and $H$ has a unique submarkovian extension by Theorem 1.1][1]. Another simple situation occurs if $c(x)=0$ for $x \leq 0$ but $c(x)>0$ for $x>0$. Then the Lipschitz condition means that $c(x)=O(x)$ as $x \rightarrow 0_{+}$and the uniqueness follows from Theorem 2.7III. To understand the general situation one needs information about the extensions of $H$ acting on a finite interval with the coefficient degenerate at both endpoints. These extensions have been extensively studied by Feller [Fel52] [Fel54 [Fel57] (see also [Man68]) for $H$ acting on the spaces $C_{b}$ and $L_{1}$ using probabilistic arguments and by Ulmet using function analytic techniques [Ulm92]. Properties of the Friedrichs extension on $L_{2}$ have also been analyzed in detail by Campiti, Metafune and Pallara [CMP98. But all self-adjoint extensions can also be studied by the methods of the previous sections. The situation for the submarkovian extensions is particularly simple.

Define $\nu(x)=\int_{x}^{1 / 2} c^{-1}$. Fix $\sigma_{0} \in C_{c}^{\infty}(0,1 / 2)$ with $0 \leq \sigma_{0} \leq 1$ and $\sigma_{0}=1$ in a neighbourhood of zero. Then set $\tau_{0}=\sigma_{0} \nu$. Further define $\sigma_{1}$ and $\tau_{1}$ as the reflections of $\sigma_{0}$ and $\tau_{0}$ around the midpoint $1 / 2$ of the interval. 
Lemma 5.1 Assume $c \in W^{1, \infty}(0,1), c>0$ on $\langle 0,1\rangle$ and $c(0)=0=c(1)$. Define the symmetric operator $H$ on $L_{2}(0,1)$ by $H \varphi=-\left(c \varphi^{\prime}\right)^{\prime}$ for $\varphi \in C_{c}^{\infty}(0,1)$.

Then $H$ has a unique submarkovian extension $H_{F}$, the Friedrichs extension, and $D\left(H_{F}\right)=$ $D(\bar{H})+\operatorname{span} \sigma_{0}+\operatorname{span} \sigma_{1}$.

Proof Since $c \in W^{1, \infty}(0,1)$ one has $c(x)=O(x)$ as $x \rightarrow 0_{+}$and $c(x)=O(1-x)$ as $x \rightarrow 1_{-}$. Therefore $\nu$ is unbounded at both endpoints 0 and 1 . Then the proof follows the arguments used for the half-line in Section 2. In fact $H_{F}\left(\varphi+a_{0} \sigma_{0}+a_{1} \sigma_{1}\right)=\bar{H} \varphi-$ $a_{0}\left(c \sigma_{0}^{\prime}\right)^{\prime}-a_{1}\left(c \sigma_{1}^{\prime}\right)^{\prime}$ for all $\varphi \in D(\bar{H})$ and $a_{0}, a_{1} \in \mathbf{R}$ in direct analogy with Remark 2.19 for the half-line and Remark 3.4 for the line.

It also follows under the assumptions of the lemma that $(I+H) C_{c}^{\infty}(0,1)$ is dense in $L_{1}(0,1)$. This can be deduced from the argument used in the proof of Lemma 4.2 or from Proposition 3.5 of [CMP98].

The foregoing lemma is the last element in the proof of Theorem 1.3.

Proof of Theorem 1.3 First, let $\mathcal{Z}=\{x \in \mathbf{R}: c(x)=0\}$ denote the zero set of the coefficient $c$. Then $L_{2}(\mathbf{R})=L_{2}(\mathcal{Z}) \oplus L_{2}\left(\mathcal{Z}^{\mathrm{c}}\right)$. Since $H=0$ in restriction to $L_{2}(\mathcal{Z})$ we only need to analyze the operator on $L_{2}\left(\mathcal{Z}^{\mathrm{c}}\right)$. Next as $c$ is continuous $\mathcal{Z}$ is closed and the complement $\mathcal{Z}^{\mathrm{c}}$ is open. Therefore $\mathcal{Z}^{\mathrm{c}}$ is the union of a family of disjoint open intervals $I_{i}$.

Secondly, $\left.H\right|_{C_{c}^{\infty}\left(I_{i}\right)}$ has a unique submarkovian extension $H_{i}$ by Theorem 2.7 if $I_{i}$ is semi-infinite and by Lemma 5.1 if $I_{i}$ is finite. In both cases $H_{i}$ is the Friedrichs extension and its action is given either by the algorithm of Remark 2.19] or by that of Lemma 5.1] In particular if $I_{i}=\left\langle a_{i}, \infty\right\rangle$ then $D\left(H_{i}\right)=D\left(\bar{H}^{i}\right)+\operatorname{span} \sigma_{a_{i}}$ where $\bar{H}^{i}$ denotes the $L_{2}\left(I_{i}\right)$ closure of $\left.H\right|_{C_{c}^{\infty}\left(I_{i}\right)}$ and $\sigma_{a_{i}}$ is a $C_{c}^{\infty}\left(I_{i}\right)$-function which is equal to one in a neighbourhood of $a_{i}$. Similarly if $I_{i}=\left\langle a_{i}, b_{i}\right\rangle$ then $D\left(H_{i}\right)=D\left(\bar{H}^{i}\right)+\operatorname{span} \sigma_{a_{i}}+\operatorname{span} \sigma_{b_{i}}$ and if $I_{i}=\left\langle-\infty, b_{i}\right\rangle$ then $D\left(H_{i}\right)=D\left(\bar{H}^{i}\right)+\operatorname{span} \sigma_{b_{i}}$.

Thirdly, set $\widetilde{H}=\bigoplus_{i} H_{i}$. Then $\widetilde{H}$ is a submarkovian extension of $\left.H\right|_{C_{c}^{\infty}\left(\mathcal{Z}^{\mathrm{c}}\right)}$ corresponding to the Friedrichs extension of $\left.H\right|_{C_{c}^{\infty}\left(\mathcal{Z}^{\mathrm{c}}\right)}$. In particular $D(\widetilde{H})=\bigoplus_{i} D\left(H_{i}\right)$ consists of the $\varphi=\bigoplus_{i} \varphi_{i}$ with $\varphi_{i} \in D\left(H_{i}\right)$ such that $\sum_{i}\left(\left\|\varphi_{i}\right\|_{L_{2}\left(I_{i}\right)}^{2}+\left\|H_{i} \varphi_{i}\right\|_{L_{2}\left(I_{i}\right)}^{2}\right)<\infty$ and then $\widetilde{H} \varphi=\bigoplus_{i} H_{i} \varphi_{i}$. Now let $\widehat{H}$ denote a second submarkovian extension of $H$. We will prove that $\left.\widehat{H}\right|_{D\left(H_{i}\right)}=H_{i}$ for each $i$ and thereby deduce that $\widehat{H} \supseteq \widetilde{H}$. But a self-adjoint operator cannot have a proper self-adjoint extension so one must have $\widehat{H}=\widetilde{H}$, i.e. $H$ has a unique submarkovian extension.

If $\varphi \in C_{c}^{\infty}\left(I_{i}\right) \subseteq D(H)$ then $\widehat{H} \varphi=H \varphi=\widetilde{H} \varphi$. Therefore $\widehat{H} \varphi=\widetilde{H} \varphi$ for all $\varphi \in D\left(\bar{H}^{i}\right)$. Next suppose $I_{i}$ has a finite left endpoint $a_{i}$ and $\sigma_{a_{i}} \in D\left(H_{i}\right)$ but $\sigma_{a_{i}} \notin D\left(\bar{H}^{i}\right)$. It follows from the proof of Proposition 4.4 (see Remark 4.6) that one may choose a sequence $\sigma_{n, i} \in C_{c}^{\infty}\left(I_{i}\right)$ such that $\left\|\sigma_{n, i}-\sigma_{a_{i}}\right\|_{1} \rightarrow 0$ and $\left\|H \sigma_{n, i}+\left(c \sigma_{a_{i}}^{\prime}\right)^{\prime}\right\|_{1} \rightarrow 0$ as $n \rightarrow \infty$. Now let $\widehat{H}_{1}$ and $\widetilde{H}_{1}$ denote the $L_{1}$-generators of the submarkovian semigroups generated by $\widehat{H}$ and $\widetilde{H}$, respectively. Then $\widehat{H}_{1} \sigma_{n, i}=H \sigma_{n, i}=\widetilde{H}_{1} \sigma_{n, i}$. But $\widehat{H}_{1}$ is $L_{1}$-closed. Therefore $\sigma_{a_{i}} \in D\left(\widehat{H}_{1}\right)$ and $\widehat{H}_{1} \sigma_{a_{i}}=-\left(c \sigma_{a_{i}}^{\prime}\right)^{\prime}$. In addition $\sigma_{a_{i}}$ and $\left(c \sigma_{a_{i}}^{\prime}\right)^{\prime}$ are both in $L_{2}\left(I_{i}\right)$. Hence $\sigma_{a_{i}} \in D\left(\widehat{H}_{1}\right) \cap L_{2}\left(I_{i}\right)$ and $\widehat{H}_{1} \sigma_{a_{i}}=-\left(c \sigma_{a_{i}}^{\prime}\right)^{\prime} \in L_{2}\left(I_{i}\right)$. Therefore $\sigma_{a_{i}} \in D(\widehat{H})$ and $\widehat{H} \sigma_{a_{i}}=\widehat{H}_{1} \sigma_{a_{i}}=-\left(c \sigma_{a_{i}}^{\prime}\right)^{\prime}$. Similarly $\sigma_{a_{i}} \in D(\widetilde{H})$ and $\widetilde{H} \sigma_{a_{i}}=-\left(c \sigma_{a_{i}}^{\prime}\right)^{\prime}$. Thus one concludes that $\widehat{H} \sigma_{a_{i}}=\widetilde{H} \sigma_{a_{i}}$. If $I_{i}$ has a finite right endpoint $b_{i}$ one concludes similarly that $\widehat{H} \sigma_{b_{i}}=\widetilde{H} \sigma_{b_{i}}$. Therefore $\widehat{H}$ and $\widetilde{H}$ are equal on $D\left(H_{i}\right)$. This completes the proof of uniqueness of the submarkovian extension. 


\section{Acknowledgement}

This project originated in a series of interesting discussions with Ricardo Weder and Gian Michele Graf whilst the first author was visiting the Institut für Theoretische Physik at the Eidgenössische Technische Hochschule, Zürich in 2007. The author is indebted to Jürg Fröhlich and Gian Michele Graf for facilitating this visit and to the ETH for providing financial support.

\section{References}

[BaR84] Batty, C. J. K., and Robinson, D. W., Positive one-parameter semigroups on ordered Banach spaces. Acta Appl. Math. 2 (1984), 221-296.

[BM95] Biroli, M., and Mosco, U., A Saint-Venant type principle for Dirichlet forms on discontinuous media. Ann. Mat. Pura Appl. 169 (1995), 125-181.

[BH91] Bouleau, N., and Hirsch, F., Dirichlet forms and analysis on Wiener space, vol. 14 of de Gruyter Studies in Mathematics. Walter de Gruyter \& Co., Berlin, 1991.

[CMP98] Campiti, M., Metafune, G., and Pallara, D., Degenerate self-adjoint evolution equations on the unit interval. Semigroup Forum 57 (1998), 1-36.

[Dav85] Davies, E. B., $L^{1}$ properties of second order elliptic operators. Bull. London Math. Soc. 17 (1985), 417-436.

[DS63] Dunford, N., and Schwartz, J. T., Linear operators. Part II: Spectral theory, self adjoint operators in Hilbert space, vol. 7 of Interscience tracts in pure and applied mathematics. Interscience Publishers, John Wiley \& Sons, New York, 1963.

[ERSZ07] Elst, A. F. M. ter, Robinson, D. W., Sikora, A., and Zhu, Y., Secondorder operators with degenerate coefficients. Proc. London Math. Soc. 95 (2007), 299-328.

[FKS82] Fabes, E. B., Kenig, C. E., and Serapioni, R. P., The local regularity of solutions of degenerate elliptic equations. Comm. Part. Diff. Eq. 7 (1982), $77-116$.

[Far75] FARIS, W. G., Self-adjoint operators. Lect. Notes in Math. 433. Springer-Verlag, Berlin etc., 1975.

[FP83] Fefferman, C., and Phong, D. H., Subelliptic eigenvalue problems. In Conference on harmonic analysis in honor of Antoni Zygmund, Wadsworth Math. Ser., 590-606. Wadsworth, Belmont, CA, 1983.

[Fel52] Feller, W., The parabolic differential equations and the associated semigroups of transformations. Ann. Math. 55 (1952), 468-519.

[Fel54] — Diffusion processes in one dimension. Trans. Amer. Math. Soc. 77 (1954), $1-31$. 
[Fel57] - Generalized second order differential operators and their lateral conditions. Illinois J. Math. 1 (1957), 459-504.

[Fra91] Franchi, B., Weighted Sobolev-Poincaré inequalities and pointwise estimates for a class of degenerate elliptic equations. Trans. Amer. Math. Soc. 327 (1991), $125-158$.

[FLW95] Franchi, B., Lu, G., and Wheeden, R. L., Weighted Poincaré inequalities for Hörmander vector fields and local regularity for a class of degenerate elliptic equations. Potential Anal. 4 (1995), 361-375.

[FOT94] Fukushima, M., Oshima, Y., and Takeda, M., Dirichlet forms and symmetric Markov processes, vol. 19 of de Gruyter Studies in Mathematics. Walter de Gruyter \& Co., Berlin, 1994.

[Kat80] Kato, T., Perturbation theory for linear operators. Second edition, Grundlehren der mathematischen Wissenschaften 132. Springer-Verlag, Berlin etc., 1980.

[MR92] MA, Z. M., and RöCKNER, M., Introduction to the theory of (non symmetric) Dirichlet Forms. Universitext. Springer-Verlag, Berlin etc., 1992.

[Man68] MandL, P., Analytical treatment of one-dimensional Markov processes. Academia Publishing House of the Czechoslovak Academy of Sciences, Prague, 1968.

[Nag86] NAGEL, R., ed., One-parameter semigroups of positive operators, Lecture Notes in Mathematics 1184, Berlin etc., 1986. Springer-Verlag.

[Pan88] PAng, M. M. H., $L_{1}$ properties of two classes of singular second order elliptic operators. J. London Math. Soc. 38 (1988), 525-543.

[PSS06] Pentry, J. B., Schurig, D., and Smith, D. R., Controlling electromagnetic fields. Science 312 (2006), 1780-1782.

[RS78] Reed, M., and Simon, B., Methods of modern mathematical physics IV. Analysis of operators. Academic Press, New York etc., 1978.

[RS07] Robinson, D. W., and Sikora, A., Degenerate elliptic operators: capacity, flux and separation. J. Ramanujan Math. Soc. 22 (2007), 385-408.

[SW06] Sawyer, E. T., and Wheeden, R. L., Hölder continuity of weak solutions to subelliptic equations with rough coefficients. Mem. Amer. Math. Soc. 180, No. 847 (2006), $\mathrm{x}+157$.

[Sto90] Stone, M., Linear transformations in Hilbert space, vol. 15 of American Mathematical Society Colloquium Publications. American Mathematical Society, Providence, RI, 1990.

[Tru73] Trudinger, N. S., Linear elliptic operators with measurable coefficients. Ann. Scuola Norm. Sup. Pisa 27 (1973), 265-308. 
[Ulm92] Ulmet, M. G., Boundary conditions for one-dimensional positive semigroups. Semigroup Forum 45 (1992), 92-119.

[Wed08] WedER, R., A rigorous analysis of high-order electromagnetic invisibility cloaks. J. Phys. A: Math. Theor. 41 (2008), 1-21. 